\title{
Multi-Enzymatic Cascades in the Synthesis of Modified Nucleosides: Comparison of the Thermophilic and Mesophilic Pathways
}

\author{
Ilja V. Fateev (D, Maria A. Kostromina, Yuliya A. Abramchik, Barbara Z. Eletskaya (D, Olga O. Mikheeva, \\ Dmitry D. Lukoshin, Evgeniy A. Zayats (D), Maria Ya. Berzina, Elena V. Dorofeeva, Alexander S. Paramonov (D, \\ Alexey L. Kayushin, Irina D. Konstantinova * and Roman S. Esipov
}

Citation: Fateev, I.V.; Kostromina, M.A.; Abramchik, Y.A.; Eletskaya, B.Z.; Mikheeva, O.O.; Lukoshin, D.D.; Zayats, E.A.; Berzina, M.Y..; Dorofeeva, E.V.; Paramonov, A.S.; et al. Multi-Enzymatic Cascades in the Synthesis of Modified

Nucleosides: Comparison of the

Thermophilic and Mesophilic Pathways. Biomolecules 2021, 11, 586. https://doi.org/10.3390/

biom 11040586

Academic Editor: Jesús Fernandez Lucas

Received: 13 January 2021

Accepted: 4 April 2021

Published: 16 April 2021

Publisher's Note: MDPI stays neutral with regard to jurisdictional claims in published maps and institutional affiliations.

Copyright: (c) 2021 by the authors Licensee MDPI, Basel, Switzerland. This article is an open access article distributed under the terms and conditions of the Creative Commons Attribution (CC BY) license (https:/ / creativecommons.org/licenses/by/ $4.0 /)$.
Shemyakin and Ovchinnikov Institute of Bioorganic Chemistry RAS, Miklukho-Maklaya 16/10, 117997 GSP, B-437 Moscow, Russia; ifateev@gmail.com (I.V.F.); kostromasha@mail.ru (M.A.K.); ugama@yandex.ru (Y.A.A.); fraubarusya@gmail.com (B.Z.E.); olga.mikheeva.92@mail.ru (O.O.M.); ldd-94@yandex.ru (D.D.L.); eaz96post@gmail.com (E.A.Z.); berzina_maria@mail.ru (M.Y.B.); iegol2013@gmail.com (E.V.D.); a.s.paramonov@gmail.com (A.S.P.); kayushin.alexej@yandex.ru (A.L.K.); esipov@ibch.ru (R.S.E.)

* Correspondence: kid1968@yandex.ru; Tel.: +7-905-791-1719

Abstract: A comparative study of the possibilities of using ribokinase $\rightarrow$ phosphopentomutase $\rightarrow$ nucleoside phosphorylase cascades in the synthesis of modified nucleosides was carried out. Recombinant phosphopentomutase from Thermus thermophilus HB27 was obtained for the first time: a strain producing a soluble form of the enzyme was created, and a method for its isolation and chromatographic purification was developed. It was shown that cascade syntheses of modified nucleosides can be carried out both by the mesophilic and thermophilic routes from D-pentoses: ribose, 2-deoxyribose, arabinose, xylose, and 2-deoxy-2-fluoroarabinose. The efficiency of 2-chloradenine nucleoside synthesis decreases in the following order: Rib (92), dRib (74), Ara (66), F-Ara (8), and Xyl $(2 \%)$ in 30 min for mesophilic enzymes. For thermophilic enzymes: Rib (76), dRib (62), Ara (32), F-Ara $(<1)$, and $\mathrm{Xyl}(2 \%)$ in $30 \mathrm{~min}$. Upon incubation of the reaction mixtures for a day, the amounts of 2-chloroadenine riboside (thermophilic cascade), 2-deoxyribosides (both cascades), and arabinoside (mesophilic cascade) decreased roughly by half. The conversion of the base to 2-fluoroarabinosides and xylosides continued to increase in both cases and reached $20-40 \%$. Four nucleosides were quantitatively produced by a cascade of enzymes from D-ribose and D-arabinose. The ribosides of 8-azaguanine (thermophilic cascade) and allopurinol (mesophilic cascade) were synthesized. For the first time, D-arabinosides of 2-chloro-6-methoxypurine and 2-fluoro-6-methoxypurine were synthesized using the mesophilic cascade. Despite the relatively small difference in temperatures when performing the cascade reactions $\left(50\right.$ and $80^{\circ} \mathrm{C}$ ), the rate of product formation in the reactions with Escherichia coli enzymes was significantly higher. E. coli enzymes also provided a higher content of the target products in the reaction mixture. Therefore, they are more appropriate for use in the polyenzymatic synthesis of modified nucleosides.

Keywords: purine nucleoside phosphorylase; biocatalysis; enzyme cascade; expression of recombinant enzymes

\section{Introduction}

Nucleoside analogs can be synthesized by chemical or enzymatic methods or by a combination of these methods [1,2]. Chemical synthesis is a long multi-stage process involving the introduction and removal of various protective groups in the carbohydrate residue and heterocyclic base, which leads to a significant decrease in the efficiency of the process. Despite the use of selective glycosylation methods, racemic mixtures are formed in the synthesis of nucleosides, which complicates the isolation of target compounds. Enzymatic synthesis has a number of advantages over chemical synthesis: mild reaction 
conditions, high stereo- and regioselectivity, the minimal use of polluting chemicals and organic solvents, high efficiency, and the absence of undesirable impurities $[1,3,4]$.

Biocatalytic reactions can involve either one enzyme that catalyzes one specific reaction or several enzymes that act sequentially in a cascade of reactions [3]. In recent years, the terms "cascade reactions" or "tandem reactions" have been used to denote poly-enzymatic reactions [5].

Bacterial glycosyltransferases that catalyze the transfer of the pentofuranosyl group to purine or pyrimidine bases are successfully used in the synthesis of various natural nucleoside analogs of biological and pharmaceutical significance. The donor of the pentofuranosyl residue can be a natural nucleoside or its derivative, while natural or modified heterocyclic bases serve as acceptors [3]. Nucleoside phosphorylases (NP) include purine nucleoside phosphorylases (PNP) and pyrimidine nucleoside phosphorylases (PyNP): uridine phosphorylases (UP) and thymidine phosphorylases (TP). One of the most studied polyenzymatic cascades in the synthesis of modified nucleosides is the sequential use of two NPs. The combination of PNP and PyNP (UP or TP) is often used in the enzymatic synthesis of nucleoside analogs. Such a combination allows obtaining purine nucleosides from pyrimidine ones and vice versa [6]. In addition, some enzymes are involved in the biosynthesis of uridyl peptide antibiotics, such as nikkomycin or tunicamycin [7]. One of the enzymes involved in the biosynthesis of the uridyl peptide antibiotic pacidamycin, dehydratase Pac13, can be used as a biocatalyst for the preparation of 3 '-deoxynucleosides [8].

The use of nucleoside phosphorylase-producing microorganisms in the synthesis of modified nucleosides of biological and pharmaceutical significance has proven to be highly effective $[9,10]$. The transglycosylation reaction is usually carried out at $60^{\circ} \mathrm{C}$ to inhibit other enzymes-for example, deaminases. At this temperature, some NPs retain most of their activity. However, reactions involving TP are usually carried out at $45^{\circ} \mathrm{C}$, since thymidine phosphorylase loses activity at temperatures above $50{ }^{\circ} \mathrm{C}$.

Temperature restrictions can be overcome by using thermophilic microorganisms such as Geobacillus stearothermophilus [11] and Thermus thermophilus [4,12,13]. Several strains of T. thermophilus that can synthesize purine nucleosides from adenine or hypoxanthine have been selected [12]. An example of pyrimidine nucleoside synthesis is the enzymatic synthesis of 5-methyluridine from inosine and thymine with the participation of immobilized PNP and PyNP Bacillus stearothermophilus JTS 859 [14]. A similar approach has been used in the synthesis of several modified nucleosides: cladribine (2-chloro- $2^{\prime}$-deoxyadenosine), fludarabine ( $2^{\prime}$-fluoradenine arabinoside), vidarabine (9- $\beta$-D-arabinosyladenine), and others. Partially purified preparations of PNP and PyNP G. stearothermophilus B-2194 immobilized on aminopropylated macroporous glass have shown high enzymatic activity and stability at $70{ }^{\circ} \mathrm{C}$ and reusability up to 20 times [15]. Immobilized thermophilic nucleoside phosphorylases have been used in the polyenzymatic synthesis of several halogenated nucleoside analogs: cladribine [4], 2-fluoro-2'-deoxyadenosine, and fludarabine [16]. The synthesis of halogenated nucleosides is of particular interest, since they exhibit a broad spectrum of biological activity $[9,17]$.

Recently published data on the activity of the hyperthermophilic PyNP from Thermus thermophilus should be noted [13]. This enzyme works in a wide $\mathrm{pH}$ range (4-9) at $100{ }^{\circ} \mathrm{C}$ and relatively high concentrations of organic solvents (up to $80 \% v / v$ DMSO and ethylene glycol).

In the case of unnatural nucleoside synthesis, the cascade of ribokinase (RK) $\rightarrow$ phosphopentomutase (PPM) $\rightarrow$ E. coli nucleoside phosphorylase (NP) appears to be promising [18]. This cascade transforms D-pentose through 5-phosphate into 1- $\alpha$-phosphate and then into the nucleoside (Scheme 1): 


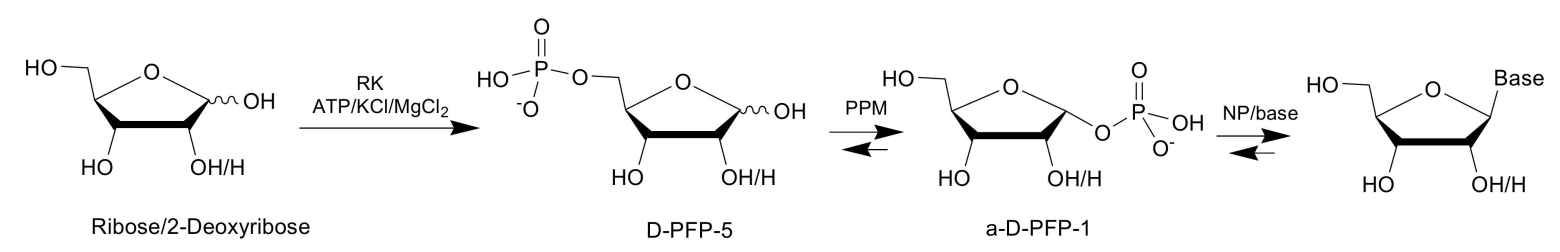

Scheme 1. One-pot enzymatic transformation of D-pentoses into nucleosides.

Phosphopentomutases catalyze the reversible transfer of the phosphate group between the $\mathrm{C} 1$ and $\mathrm{C} 5$ atoms of ribose or deoxyribose [19]. The resulting $\alpha$-D-ribose-1phosphate is a substrate for nucleoside phosphorylases in the synthesis of nucleosides. Tandem enzymatic cascades of nucleoside synthesis using two enzymes (E. coli PPM and NP combination) have been adapted for the synthesis of D-ribose, D-arabinose, and 2deoxy-D-ribose nucleosides $[18,20]$. The possibility of the polyenzymatic synthesis of 2 -deoxy- $\beta$-D-ribofuranosides-8-aza-purine and 8-aza-7-deazapurine from 2-deoxyribose using ribokinase, PPM, and NP has been shown [21].

Cascades of thermophilic microorganism enzymes can also be used for the synthesis of modified nucleotides. Therefore, in 2016, a cascade of thermophilic enzyme Thermus species 2.9 was presented for the synthesis of nucleotides from D-pentoses [22].

For the thermophilic cascade synthesis of nucleosides, we previously obtained and described ribokinase from Thermus species 2.9 (TspRK, gene QT17_05185) [23], purine nucleoside phosphorylases from Thermus thermophilus HB27 (TthPNPI, gene TT_RS05405, and TthPNPII, gene TT_RS00985) [24].

The synthesis of natural nucleosides using various nucleoside phosphorylases yields similar results. However, with modified heterocyclic bases or different carbohydrates, the results may vary significantly. It would be promising to obtain recombinant TthPPM and try the complete cascade of thermophilic RK $\rightarrow$ PPM $\rightarrow$ PNP to synthesize modified nucleosides. It is essential to carry out a comparative study of the features and efficiency of the cascade syntheses of nucleosides using mesophilic and thermophilic enzymes. In addition, some heterocyclic bases, such as 2-chloroadenine and 2-fluoroadenine, have low water solubility, so the use of thermophilic enzymes might be preferable. Carrying out the reaction with thermophilic enzymes at the operating temperatures of mesophilic enzymes reduces the benefits of this approach; therefore, the reactions with Thermus thermophilus enzymes were carried out at a higher temperature.

\section{Materials and Methods}

\subsection{General Procedures}

Tris $\cdot \mathrm{HCl}$, acetic acid, sodium chloride, glycerol, acrylamide, N, $\mathrm{N}^{\prime}$-bisacrylamide, ATP disodium salt trihydrate, bromophenol blue, agarose, ethylenediaminetetraacetic acid (EDTA), isopropyl $\beta$-D-1-thiogalactopyranoside (IPTG), imidazole, and dimethylformamide (DMF) were purchased from Panreac (Barcelona, Spain). Ethanol was purchased from MedChemProm (Balashikha, Russia). Coomassie Brilliant Blue R-250 was purchased from Bio-Rad. Bacto yeast extract, bacto tryptone, and bacto agar were purchased from Becton Dickinson Biosciences (Franklin Lakes, NJ, USA). $\mathrm{NaOH}, \mathrm{HCl}$, pyridine, $\mathrm{POCl} 3$, Et2NPh, and hydrogen fluoride pyridine (HF/Py) $(70 \%)$ were purchased from Merck (Darmstadt, Germany). Sodium persulfate, tetramethylethylenediamine (TEMED), ethidium bromide, and sodium azide were purchased from Helicon (Moscow, Russia). deoxynucleoside triphosphate mix (dNTP) was purchased from Fermentas (Waltham, MA, USA). Dithiothreitol (DTT), phenylmethylsulfonylchloride, magnesium chloride, nickel sulfate, potassium dihydroorthophosphate, 8-azaguanine, allopurinol, 2-chloroadenin, tert-butyl nitrite, and Ni-IDA Sepharose were purchased from Sigma-Aldrich (St. Louis, MO, USA). Sodium dodecylsulfate was purchased from Serva (Heidelberg, Germany). Ampicillin was purchased from AppliChem GmbH (Darmstadt, Germany). Polyethersulfone membranes were purchased from Millipore (Burlington, MA, USA). 
Unless otherwise noted, the materials were obtained from commercial suppliers and used without any purification.

9-(2,3,5-Tri-O-acetyl- $\beta$-D-ribofuranosyl)-2,6-dichloropurine was synthesized as described in $[25,26]$. 9-(2,3,5-Tri-O-acetyl- $\beta$-D-ribofuranosyl)-2-amino-6-chloropurine was synthesized as described in [27].

NMR spectra were recorded on Bruker Avance II 700 spectrometers (Bruker BioSpin, Rheinstetten, Germany) in DMSO-d6 at $303 \mathrm{~K}$. Chemical shifts in ppm $(\delta)$ were measured relative to the residual solvent signals as internal standards (2.508 ppm). Coupling constants (J) were measured in $\mathrm{Hz}$; s—singlet, br.s.—-broad signal, d-doublet, $\mathrm{m}$-multiplet, $\mathrm{t}$ - triplet. NMR spectra data were provided in the Supplementary Materials.

Liquid chromatography-mass spectrometry was performed on the Agilent $6210 \mathrm{TOF}$ LC/MS system (Agilent Technologies, Santa Clara, CA, USA).

UV spectra were recorded on a Hitachi U-2900 spectrophotometer (Hitachi, Tokyo, Japan).

\subsection{Purification of Recombinant Enzymes}

In this study, the following previously obtained recombinant enzymes were used: ribokinase from Escherichia coli (EcRK, gene AAA51476) [18,28], phosphopentomutase from E. coli (EcPPM, gene AAA97279) [18], purine nucleoside phosphorylase from E. coli (EcPNP, gene AAA24401) [29], ribokinase from Thermus species 2.9 (TspRK, gene QT17_05185) [23], purine nucleoside phosphorylases from Thermus thermophilus HB27 (TthPNPI, gene TT_ RS05405, and TthPNPII, gene TT_RS00985) [24]. Using the protocols described in their respective publications, batches of purified enzymes were prepared in quantities sufficient for all the experiments.

\subsection{Cloning, Expression, and Purification of Recombinant TthPPM}

The TT_RS08405 gene encoding the phosphopentomutase from Thermus thermophilus HB27 was amplified from genomic DNA by PCR with primer PPM-forward (5'-GGTGGTC ATATGAAGGCGGTGGCCATCGTTTTG-3') and PPM-reverse (5'-GGTGGTGCGGCCGCG ACGAGGCTCGTTCCGGGG-3') and cloned into the pET-23a+ expression vector at the NdeI and NotI restriction sites. The resulting plasmid pER-PPM-Tth contained the gene encoding the TthPPM with the C-terminal His-tag. The producing strain E. coli NiCo21 (DE3)/pER-PPM-Tth was obtained. Cultivation of the strain was carried out in a lysogeny broth (LB) medium containing $100 \mu \mathrm{g} / \mathrm{mL}$ ampicillin. After reaching an absorbance of $\mathrm{A}_{595}=0.8$, the cultures were added with IPTG to a final concentration of $0.4 \mathrm{mM}$, and cultivation was continued for $4 \mathrm{~h}$ at $37^{\circ} \mathrm{C}$. After culturing, the cell biomass was separated by centrifugation ( $2.8 \mathrm{~g}$ of wet biomass per liter).

The cell biomass (5.6 g) was disrupted in $50 \mathrm{mM} \mathrm{KH}_{2} \mathrm{PO}_{4}, \mathrm{pH}$ 6.8, $10 \mathrm{mM}$ EDTA, $1 \mathrm{mM}$ phenylmethylsulphonyl fluoride (PMSF) in a ratio of 1:10 $(w / v)$ using an ultrasonicator. The clarified cell lysate was heat-treated at $80{ }^{\circ} \mathrm{C}$ for $10 \mathrm{~min}$ to precipitate the contaminated proteins and DNA. To purify TthPPM, a two-stage technique was developed. In the first step, the protein was purified by anion exchange chromatography using an XK 16/20 column packed with $10 \mathrm{~mL}$ of DEAE Sepharose Fast Flow resin and equilibrated with $10 \mathrm{mM} \mathrm{KH} \mathrm{PO}_{4}$ and $1 \mathrm{mM}$ PMSF, $\mathrm{pH}$ 6.8. The target protein was eluted with a linear gradient from 0- to $0.4-\mathrm{M} \mathrm{NaCl}(100 \mathrm{~mL}, 2 \mathrm{~mL} / \mathrm{min})$. The chromatography eluate was loaded onto an XK 16/20 column packed with $20 \mathrm{~mL}$ of Chelating Sepharose Fast Flow and equilibrated with $50 \mathrm{mM}$ Tris $\cdot \mathrm{HCl}, \mathrm{pH}$ 8.5. The second chromatography was performed on an XK 16/20 column packed with $20 \mathrm{~mL}$ of Chelating Sepharose Fast Flow and equilibrated with $50 \mathrm{mM}$ Tris $\cdot \mathrm{HCl}, \mathrm{pH} 8.5$, and $5 \mathrm{mM}$ EDTA in a linear gradient of 50-200 mM imidazole (100 mL, $2 \mathrm{~mL} / \mathrm{min})$. The eluate was concentrated using a 30-kDa cut-off polyethersulfone membrane and then loaded on a HiLoad 16/60 Superdex 75-g size-exclusion column equilibrated with $20 \mathrm{mM}$ Tris. $\mathrm{HCl}$, pH 8.0, supplemented with $50 \mathrm{mM} \mathrm{NaCl}, 5 \%$ glycerol, and $0.04 \%(w / v) \mathrm{NaN}_{3}$. Fractions containing a target enzyme with purity higher than $90 \%$ were combined and concentrated to the final concentration of $10.0 \pm 0.5 \mathrm{mg} / \mathrm{mL}$. 


\subsection{Analytical Methods}

The protein concentration was determined by the Lowry method, using bovine serum albumin (BSA) as the standard [30]. Protein purity was determined using protein electrophoresis in polyacrylamide gel under denaturing conditions [31].

Oligomeric organization of enzymes was assessed by size-exclusion chromatography (SEC) using a Superdex 200 10/300 GL analytical column (GE Healthcare, USA). The analysis parameters were as follows: eluent $25 \mathrm{mM} \mathrm{Na}_{2} \mathrm{HPO}_{4}, \mathrm{pH} 8.0,150 \mathrm{mM} \mathrm{NaCl}$, and $0.04 \%(w / v) \mathrm{NaN}_{3}$; flow rate $0.6 \mathrm{~mL} / \mathrm{min}$; time $60 \mathrm{~min}$; detection $220 \mathrm{~nm}$; oven temperature $23^{\circ} \mathrm{C}$; and sample injection volume is $100 \mu \mathrm{L}(50 \mu \mathrm{g})$. Gel Filtration Markers MWGF200 and MWGF1000 (Sigma-Aldrich, St. Louis, MO, USA) were used for plotting the calibration curve and calculating the molecular weight.

The analysis of the efficiency of nucleoside synthesis cascades was carried out using the following chromatographic systems:

HPLC system 1: Hitachi Chromaster, column YMC Triart-C18, 50×3.0 mm, $3 \mu \mathrm{m}$, eluent $0.1 \%$ aqueous trifluoroacetic acid (TFA), detection at $254 \mathrm{~nm}$, and flow rate $0.4 \mathrm{~mL} / \mathrm{min}$.

HPLC system 2: Hitachi Chromaster, column Supelcosil LC-18-T, $150 \times 4.6 \mathrm{~mm}, 5 \mu \mathrm{m}$, eluent $0.1 \mathrm{M} \mathrm{KH}_{2} \mathrm{PO}_{4} \mathrm{pH} 6.0$, detection at $254 \mathrm{~nm}$, and flow rate $0.4 \mathrm{~mL} / \mathrm{min}$.

HPLC system 3: Waters system (Waters 1525, Waters 2489, Breeze 2), column Nova Pak $\mathrm{C}_{18}, 4.6 \times 150 \mathrm{~mm}, 5 \mu \mathrm{m}$, and flow rate $0.5 \mathrm{~mL} / \mathrm{min}$. Eluent A: $0.1 \% \mathrm{TFA} / \mathrm{H}_{2} \mathrm{O}$ and eluent $\mathrm{B}: 70 \% \mathrm{MeCN}$ in $0.1 \%$ TFA $/ \mathrm{H}_{2} \mathrm{O}$, gradient $0-50 \% \mathrm{~B}, 20 \mathrm{~min}$, detection at $254 \mathrm{~nm}$.

HPLC system 4: Waters system (Waters 1525 , Waters 2489 , Breeze 2), column Nova Pak $\mathrm{C}_{18}, 4.6 \times 150 \mathrm{~mm}, 5 \mu \mathrm{m}$, and flow rate $1.0 \mathrm{~mL} / \mathrm{min}$. Eluent A: $0.1 \% \mathrm{TFA} / \mathrm{H}_{2} \mathrm{O}$ and eluent $\mathrm{B}: 70 \% \mathrm{MeCN}$ in $0.1 \%$ TFA $/ \mathrm{H}_{2} \mathrm{O}$, gradient $0-50 \% \mathrm{~B}, 20 \mathrm{~min}$, detection at $254 \mathrm{~nm}$.

HPLC system 5: Waters system (Waters 1525, Waters 2489, Breeze), column Nova Pak $\mathrm{C}_{18}, 4.6 \times 150 \mathrm{~mm}, 5 \mu \mathrm{m}$, and flow rate $1.0 \mathrm{~mL} / \mathrm{min}$. Eluent A: $0.1 \% \mathrm{TFA} / \mathrm{H}_{2} \mathrm{O}$ and eluent $\mathrm{B}$ : $70 \% \mathrm{MeCN}$ in $0.1 \% \mathrm{TFA} / \mathrm{H}_{2} \mathrm{O}$, gradient $0-100 \% \mathrm{~B}, 20 \mathrm{~min}$, detection at $254 \mathrm{~nm}$.

\subsection{Enzyme Assay}

Phosphopentomutase Thermus thermophilus HB27 (TthPPM). Each reaction mixture ( $0.25 \mathrm{~mL}, 20 \mathrm{mM}$ Tris $\cdot \mathrm{HCl}, \mathrm{pH} 8.0$ ) contained $0.5 \mathrm{mM}$ D-ribose 5-phosphate, $0.5 \mathrm{mM}$ adenine, $0.5 \mathrm{mM} \mathrm{MnCl} 2,0.2 \mu \mathrm{g}$ TthPPM, and $1.4 \mu \mathrm{g}$ TthPNPII. The reaction mixtures were incubated at $80^{\circ} \mathrm{C}$. Substrate and product quantities were determined using HPLC system 1.

Phosphopentomutase E. coli (EcPPM). Each reaction mixture $(0.25 \mathrm{~mL}, 20 \mathrm{mM}$ Tris $\cdot \mathrm{HCl}$, $\mathrm{pH}$ 7.5) contained 0.5 mM D-ribose 5-phosphate, $0.5 \mathrm{mM}$ adenine, $0.1 \mathrm{mM} \mathrm{MnCl} 2,0.26 \mu \mathrm{g}$ EcPPM, and $1.1 \mu \mathrm{g} E c$ PNP. The reaction mixtures were incubated at $37^{\circ} \mathrm{C}$. Substrate and product quantities were determined using HPLC system 1.

Purine nucleoside phosphorylase I Thermus thermophilus HB27 (TthPNPI). Each reaction mixture $\left(0.25 \mathrm{~mL}, 25 \mathrm{mM} \mathrm{KH}_{2} \mathrm{PO}_{4}, \mathrm{pH} 8.0\right)$ contained $1 \mathrm{mM}$ inosine and $0.025 \mu \mathrm{g}$ TthPNPI. The reaction mixtures were incubated at $80{ }^{\circ} \mathrm{C}$. Substrate and product quantities were determined using HPLC system 1.

Purine nucleoside phosphorylase II Thermus thermophilus HB27 (TthPNPII). Each reaction mixture $\left(0.25 \mathrm{~mL}, 25 \mathrm{mM} \mathrm{KH}_{2} \mathrm{PO}_{4}, \mathrm{pH} 8.0\right)$ contained $1 \mathrm{mM}$ adenosine and $0.03 \mu \mathrm{g}$ TthPNPII. The reaction mixtures were incubated at $80^{\circ} \mathrm{C}$. Substrate and product quantities were determined using HPLC system 1.

Purine nucleoside phosphorylase E. coli (EcPNP). Each reaction mixture $(0.25 \mathrm{~mL}, 25 \mathrm{mM}$ $\mathrm{KH}_{2} \mathrm{PO}_{4}, \mathrm{pH}$ 7.0) contained $1 \mathrm{mM}$ inosine and $0.11 \mu \mathrm{g} E c \mathrm{PNP}$. The reaction mixtures were incubated at $37^{\circ} \mathrm{C}$. Substrate and product quantities were determined using HPLC system 1.

Ribokinase Thermus species 2.9 (TspRK). Each reaction mixture $(0.25 \mathrm{~mL}, 20 \mathrm{mM}$ Tris. $\mathrm{HCl}$, pH 8.0) contained $0.4 \mathrm{mM}$ ATP, $1.2 \mathrm{mM}$ D-ribose, $5 \mathrm{mM} \mathrm{MgCl} 2,50 \mathrm{mM} \mathrm{KCl}$, and $0.3 \mu \mathrm{g}$ TspRK. The reaction mixtures were incubated at $80^{\circ} \mathrm{C}$. Substrate and product quantities were determined using HPLC system 2.

Ribokinase E. coli (EcRK). Each reaction mixture $(0.25 \mathrm{~mL}, 20 \mathrm{mM}$ Tris. $\mathrm{HCl}, \mathrm{pH} 8.0)$ contained $0.4 \mathrm{mM}$ ATP, $1.2 \mathrm{mM}$ D-ribose, $5 \mathrm{mM} \mathrm{MgCl}_{2}, 50 \mathrm{mM} \mathrm{KCl}$, and $0.02 \mu \mathrm{g}$ EcRK. 
The reaction mixtures were incubated at $37^{\circ} \mathrm{C}$. Substrate and product quantities were determined using HPLC system 2.

2.6. Determination of Kinetic Parameters of Phosphopentomutase Thermus Thermophilus HB27 (TthPPM)

Each reaction mixture $(100 \mu \mathrm{L}, 20 \mathrm{mM}$ Tris $\mathrm{HCl}, \mathrm{pH}$ 8.0) contained $1 \mathrm{mM}$ adenine, $0.5 \mathrm{mM} \mathrm{MnCl}_{2}$, and $0.25 \mu \mathrm{M} \alpha$-D-glucose 1,6-bisphosphate, from 0.011 to $1.8 \mathrm{mM} D$-ribose 5-phosphate or 2-deoxy-D-ribose 5-phosphate, 0.02 or $0.05 \mu \mathrm{g}$ TthPPM, and $0.5 \mu \mathrm{g}$ TthPNPII. The reaction mixtures were incubated $2 \mathrm{~min}$ at $80^{\circ} \mathrm{C}$. HPLC system 1 . Each experiment was repeated three times. Kinetic parameters were determined by nonlinear regression analysis using SciDAVis v2.3.0 software. Catalytic constants $\left(\mathrm{k}_{\mathrm{cat}}\right)$ were calculated per 1 subunit ( $43.2 \mathrm{kDa}$, calculated based on amino acid sequences).

\subsection{Cascade Reactions}

Testing of D-pentoses. Each reaction mixture $(0.5 \mathrm{~mL}, 20 \mathrm{mM}$ Tris $\cdot \mathrm{HCl}, \mathrm{pH} 8.0)$ contained $D$-pentose (2 mM D-ribose or 2-deoxy-D-ribose or $60 \mathrm{mM}$ D-arabinose or D-xylose or $5 \mathrm{mM}$ 2-deoxy-2-fluoro-D-arabinose); $0.34 \mathrm{mM}$ 2-chloroadenine; $2.5 \mathrm{mM}$ ATP; $2.5 \mathrm{mM}$ $\mathrm{MnCl}_{2} ; 50 \mathrm{mM} \mathrm{KCl} ; 2 \mathrm{mM} \mathrm{KH}{ }_{2} \mathrm{PO}_{4}$; and thermophilic or mesophilic recombinant enzymes (ribokinase, phosphopentomutase, and purine nucleoside phosphorylase). The reaction mixtures were incubated at 80 or $50^{\circ} \mathrm{C}$. Substrate and product quantities were determined using HPLC system 1.

Testing of heterocyclic bases. Each reaction mixture $(0.5 \mathrm{~mL}, 20 \mathrm{mM}$ Tris. $\mathrm{HCl}, \mathrm{pH} 8.0)$ contained $2 \mathrm{mM}$ D-ribose, $0.5 \mathrm{mM}$ heterocyclic base (allopurinol or 8-azaguanine), $2.5 \mathrm{mM}$ ATP, $2.5 \mathrm{mM} \mathrm{MnCl}_{2}, 50 \mathrm{mM} \mathrm{KCl}, 2 \mathrm{mM} \mathrm{KH} \mathrm{PO}_{4}$, and thermophilic or mesophilic recombinant enzymes (ribokinase, phosphopentomutase, and purine nucleoside phosphorylase). The reaction mixtures were incubated at 80 or $50{ }^{\circ} \mathrm{C}$. Substrate and product quantities were determined using HPLC system 1.

\subsection{Nucleosides Synthesis}

2.8.1. 2-Chloro-6-methoxy-9-( $\beta$-D-ribofuranosyl)purine (2)

To a solution of MeONa in $\mathrm{MeOH}(376 \mathrm{mg}, 16.2 \mathrm{mmol} \mathrm{Na}$ in $8 \mathrm{~mL}$ of $\mathrm{MeOH}), 1.0 \mathrm{~g}$ (2.2 $\mathrm{mmol}$ ) of dichloride (1) was added at room temperature under stirring. In $2 \mathrm{~h}$, the reaction mixture was cooled up to $0{ }^{\circ} \mathrm{C}$, and conc. $\mathrm{HCl}$ was added up to $\mathrm{pH} 7.0 . \mathrm{NaCl}$ was filtered off, and the precipitate was washed by hot $\mathrm{MeOH}(3 \times 3 \mathrm{~mL})$. The filtrate was evaporated to a quarter of the volume, $1 \mathrm{~mL}$ of $\mathrm{H}_{2} \mathrm{O}$ was added, and the solution was heated to boiling. The clear solution was left at room temperature. The crystals were filtered off and washed with $\mathrm{iPrOH}$ and $\mathrm{Et}_{2} \mathrm{O}$. Yield $560 \mathrm{mg}$, purity $74 \%$. The desired product was purified by chromatography on silica gel $(3 \times 30 \mathrm{~cm}$, elution by gradient of $\mathrm{MeOH}$ in $\mathrm{CHCl}_{3}(0-20 \%), 1 \mathrm{~L}$, with a flow rate of $6 \mathrm{~mL} / \mathrm{min}$. Yield $310 \mathrm{mg}(0.97 \mathrm{mmol}$, $44 \%$ ), purity $92.9 \%$ (HPLC system 5 ).

HRMS (ESI+): $m / z[\mathrm{M}+\mathrm{H}]^{+}$calc. for $\mathrm{C}_{11} \mathrm{H}_{14} \mathrm{O}_{5} \mathrm{~N}_{4} \mathrm{Cl} 317.0654 / 319.0624 \mathrm{Cl}^{35} / \mathrm{Cl}^{37}$, found 317.0636/319.0609, and [Base $+\mathrm{H}^{+}$calc. for $\mathrm{C}_{6} \mathrm{H}_{6} \mathrm{~N}_{4} \mathrm{OCl} 185.0230 / 187.0200 \mathrm{Cl}^{35}$ / $\mathrm{Cl}^{37}$, found $185.0221 / 187.0189$.

${ }^{1} \mathrm{H}$ NMR $(700 \mathrm{MHz}): \delta=8.67(\mathrm{~s}, 1 \mathrm{H}, \mathrm{H}-8), 5.93\left(\mathrm{~d}, \mathrm{~J}_{\mathrm{H} 1, \mathrm{H} 2}=5.5,1 \mathrm{H}, \mathrm{H}-1^{\prime}\right), 5.64$ and 5.35 (br. s, $2 \mathrm{H}, \mathrm{OH}-2^{\prime}$, and $\left.\mathrm{OH}-3^{\prime}\right), 5.06\left(\mathrm{~m}, 1 \mathrm{H}, \mathrm{OH}-5^{\prime}\right), 4.52\left(\mathrm{t}, J=5.1,1 \mathrm{H}, \mathrm{H}-2^{\prime}\right), 4.16(\mathrm{t}, J=4.2$, $\left.1 \mathrm{H}, \mathrm{H}-3^{\prime}\right), 4.12$ (s, $\left.3 \mathrm{H}, \mathrm{OMe}\right), 3.98\left(\mathrm{~m}, 1 \mathrm{H}, \mathrm{H}-4^{\prime}\right), 3.68\left(\mathrm{dd}, J=4.0,12.2,1 \mathrm{H}, \mathrm{H}-5^{\prime} \mathrm{a}\right), 3.58$ $\left(\mathrm{dd}, J=3.7,12.0,1 \mathrm{H}, \mathrm{H}-5^{\prime} \mathrm{b}\right) .{ }^{13} \mathrm{C}$ NMR $(176 \mathrm{MHz}): \delta=160.78$ (C-6), 152.84 (C-4), 151.43 (C-2), $142.72(\mathrm{C}-8), 120.19(\mathrm{C}-5), 87.59\left(\mathrm{C}-1^{\prime}\right), 85.63\left(\mathrm{C}-4^{\prime}\right), 73.85\left(\mathrm{C}-2^{\prime}\right), 70.05\left(\mathrm{C}-3^{\prime}\right), 61.00$ $\left(\mathrm{C}-5^{\prime}\right), 54.89\left(\mathrm{OCH}_{3}\right) .{ }^{15} \mathrm{~N}$ NMR $(71 \mathrm{MHz}): \delta=241.3(\mathrm{~N}-7), 172.47(\mathrm{~N}-9)$.

\subsubsection{2-Fluoro-6-chloro-9-(2',3,'5'-tri-O-acetyl- $\beta$-D-ribofuranosyl)purine (5)}

A $70 \%$ solution of $\mathrm{HF}$ in pyridine $(7.5 \mathrm{~mL})$ was cooled to $-18{ }^{\circ} \mathrm{C}$, and $3.20 \mathrm{~g}(7.4 \mathrm{mmol})$ of monochloride (4) was added. Some tert-butyl $(2.15 \mathrm{~mL})(28.5 \mathrm{mmol})$ was added dropwise within $8 \mathrm{~min}$, and the reaction mixture was stirred at $-18{ }^{\circ} \mathrm{C}$ for $30 \mathrm{~min}$ and at room 
temperature for $20 \mathrm{~min}$. The mixture was poured into a glass with ice $(150 \mathrm{~g})$ and $\mathrm{CaCO}_{3}$ (15 g), filtered off, and the precipitate was washed by $\mathrm{CHCl}_{3}(150 \mathrm{~mL})$. The organic layer was washed by water $(100 \mathrm{~mL}), 5 \% \mathrm{NaHCO}_{3}(100 \mathrm{~mL})$, water $(150 \mathrm{~mL})$, dried by $\mathrm{MgSO}_{4}$, and concentrated. The desired product was isolated by column chromatography on silica gel $(2 \times 9 \mathrm{~cm} ; 7 \mathrm{~cm}$ of silica gel covered by $2 \mathrm{~cm}$ of activated carbon, elution by $200 \mathrm{~mL}$ of $\mathrm{CHCl}_{3}$, then by $5 \% \mathrm{MeOH}$ in $\mathrm{CHCl}_{3}$, with a flow rate $\left.0.9 \mathrm{~mL} / \mathrm{min}\right)$. Yield $1.49 \mathrm{~g}(3.4 \mathrm{mmol}$, $46 \%$ ), purity $88.7 \%$ (HPLC system 5 ).

HRMS (ESI+): $m / z[\mathrm{M}+\mathrm{H}]^{+}$calc. for $\mathrm{C}_{16} \mathrm{H}_{17} \mathrm{O}_{7} \mathrm{~N}_{4} \mathrm{FCl} 431.0764 / 433.0735 \mathrm{Cl}^{35} / \mathrm{Cl}^{37}$, found $431.0766 / 433.0736$.

${ }^{1} \mathrm{H}$ NMR $(700 \mathrm{MHz}): \delta=8.90(\mathrm{~s}, 1 \mathrm{H}, \mathrm{H}-8), 6.31\left(\mathrm{~d}, J=5.2,1 \mathrm{H}, \mathrm{H}-1^{\prime}\right), 5.92(\mathrm{t}, J=5.5$, $\left.1 \mathrm{H}, \mathrm{H}-2^{\prime}\right), 5.61\left(\mathrm{t}, J=5.6,1 \mathrm{H}, \mathrm{H}-3^{\prime}\right), 4.44\left(\mathrm{~m}, 1 \mathrm{H}, \mathrm{H}-4^{\prime}\right), 4.40\left(\mathrm{dd}, J=3.4,12.2,1 \mathrm{H}, \mathrm{H}-5^{\prime} \mathrm{a}\right)$,

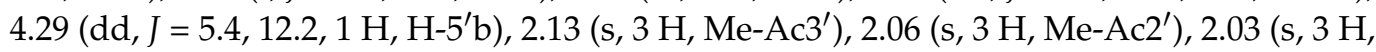
Me-Ac5 $\left.5^{\prime}\right) .{ }^{13} \mathrm{C}$ NMR $(176 \mathrm{MHz}): \delta=169.88\left(\mathrm{C}=\mathrm{O}_{\mathrm{Ac} 5^{\prime}}\right), 169.24\left(\mathrm{C}=\mathrm{O}_{\mathrm{Ac} 3^{\prime}}\right), 169.09\left(\mathrm{C}=\mathrm{O}_{\mathrm{Ac}^{\prime}}\right)$, $156.01\left(\mathrm{~d}, \mathrm{C}-2, J_{\mathrm{C} 2, \mathrm{~F} 2}=215.1\right), 153.12\left(\mathrm{~d}, \mathrm{C}-4, J_{\mathrm{C} 4, \mathrm{~F} 2}=16.6\right), 150.95\left(\mathrm{~d}, \mathrm{C}-6, J_{\mathrm{C} 6, \mathrm{~F} 2}=17.3\right)$, 146.79 (C-8), 130.65 (C-5), $86.09\left(\mathrm{C}-1^{\prime}\right), 79.70\left(\mathrm{C}-4^{\prime}\right), 72.01\left(\mathrm{C}-2^{\prime}\right), 69.63\left(\mathrm{C}-3^{\prime}\right), 62.51\left(\mathrm{C}-5^{\prime}\right)$, $20.29\left(\mathrm{CMe}_{\mathrm{Ac}^{\prime}}\right), 20.22\left(\mathrm{CMe}_{\mathrm{Ac}^{\prime}}\right), 20.07\left(\mathrm{CMe}_{\mathrm{Ac}^{\prime}}\right) .{ }^{15} \mathrm{~N} \mathrm{NMR}(71 \mathrm{MHz}): \delta=243.03(\mathrm{~N}-7)$, $169.79(\mathrm{~N}-9)$.

\subsubsection{2-Fluoro-6-methoxy-9-( $\beta$-D-ribofuranosyl)purine (6)}

To a solution of MeONa in $\mathrm{MeOH}(376 \mathrm{mg}, 16.2 \mathrm{mmol} \mathrm{Na}$ in $8 \mathrm{~mL}$ of $\mathrm{MeOH}), 1.0 \mathrm{~g}$ (2.3 mmol) of nucleoside (5) was added at room temperature under stirring. In $2 \mathrm{~h}$, the reaction mixture was cooled up to $0^{\circ} \mathrm{C}$, and conc. $\mathrm{HCl}$ was added up to $\mathrm{pH} 7.0$. $\mathrm{NaCl}$ was filtered off, and the precipitate was washed by hot $\mathrm{MeOH}(3 \times 3 \mathrm{~mL})$ and concentrated. The desired product was isolated by column chromatography on silica gel $(4.6 \times 40 \mathrm{~cm}$, elution by a gradient of $\mathrm{MeOH}$ in $\mathrm{CHCl}_{3}(0-30 \%, 1 \mathrm{~L}$, flow rate $6 \mathrm{~mL} / \mathrm{min})$. Yield $358 \mathrm{mg}$ (1.2 mmol, 50\%), purity $97.0 \%$ (HPLC system 5 ).

HRMS (ESI+): $m / z$ [M + H] $]^{+}$calc. for $\mathrm{C}_{11} \mathrm{H}_{14} \mathrm{O}_{5} \mathrm{~N}_{4} \mathrm{~F} 301.0949$, found 301.0906, [Base + $\mathrm{H}^{+}$calc. for $\mathrm{C}_{6} \mathrm{H}_{6} \mathrm{~N}_{4} \mathrm{OF} 169.0525$, found 169.0504 .

${ }^{1} \mathrm{H}$ NMR $(700 \mathrm{MHz}): \delta=8.63(\mathrm{~s}, 1 \mathrm{H}, \mathrm{H}-8), 5.89\left(\mathrm{~d}, \mathrm{~J}_{\mathrm{H} 1, \mathrm{H} 2}=5.5,1 \mathrm{H}, \mathrm{H}-1^{\prime}\right), 4.52(\mathrm{t}$, $\left.J=5.3,1 \mathrm{H}, \mathrm{H}-2^{\prime}\right), 4.16\left(\mathrm{t}, J=4.3,1 \mathrm{H}, \mathrm{H}-3^{\prime}\right), 4.13(\mathrm{~s}, 3 \mathrm{H}, \mathrm{OMe}), 3.97\left(\mathrm{~m}, 1 \mathrm{H}, \mathrm{H}-4^{\prime}\right), 3.68(\mathrm{dd}$, $\left.J=4.1,12.0,1 \mathrm{H}, \mathrm{H}-5^{\prime} \mathrm{a}\right), 3.57\left(\mathrm{dd}, J=4.1,12.0,1 \mathrm{H}, \mathrm{H}-5^{\prime} \mathrm{b}\right) .{ }^{13} \mathrm{C}$ NMR $(176 \mathrm{MHz}): \delta=162.16$ $\left(\mathrm{d}, J_{C 6, F}=18.0, C-6\right), 156.97\left(\mathrm{~d}, J_{C 2, F}=210.5, C-2\right), 152.89\left(\mathrm{~d}, J_{C 4, F}=18.8, \mathrm{C}-4\right), 142.53\left(\mathrm{~d}, J_{C 8, F}\right.$ $=2.0, \mathrm{C}-8), 119.35\left(\mathrm{~d}, J_{C 5, F}=5.0, \mathrm{C}-5\right), 87.57\left(\mathrm{C}-1^{\prime}\right), 85.54\left(\mathrm{C}-4^{\prime}\right), 73.73\left(\mathrm{C}-2^{\prime}\right), 70.01\left(\mathrm{C}-3^{\prime}\right)$, $60.98\left(\mathrm{C}-5^{\prime}\right), 54.96\left(\mathrm{OCH}_{3}\right) .{ }^{15} \mathrm{~N}$ NMR $(71 \mathrm{MHz}): \delta=241.76(\mathrm{~N}-7), 172.78(\mathrm{~N}-9)$.

\subsubsection{5-Amino-3-( $\beta$-D-ribofuranosyl)triazolo [4,5-d]pyrimidin-7-one (8)}

8-Azaguanine (28 mg, $0.184 \mathrm{mmol})$, D-ribose (42.6 mg, $0.284 \mathrm{mmol}), \mathrm{MnCl}_{2}(44.7 \mathrm{mg}$, $0.355 \mathrm{mmol}), \mathrm{KCl}(529 \mathrm{mg}, 7.1 \mathrm{mmol})$, and $\mathrm{KH}_{2} \mathrm{PO}_{4}(38.6 \mathrm{mg}, 0.284 \mathrm{mmol})$ were dissolved in Tris. $\mathrm{HCl}(20 \mathrm{mM}, 142 \mathrm{~mL}, \mathrm{pH} 8.0)$ under stirring and heating at $80^{\circ} \mathrm{C}$. ATP disodium salt trihydrate $(215 \mathrm{mg}, 0.355 \mathrm{mmol}), T \mathrm{spRK}(0.6 \mathrm{mg}, 11.4$ units, concentration in the reaction mixture $4.2 \mu \mathrm{g} / \mathrm{mL})$, TthPPM $(0.2 \mathrm{mg}, 2.8$ units, concentration in the reaction mixture $1.4 \mu \mathrm{g} / \mathrm{mL})$, and ThPNPI $(0.063 \mathrm{mg}, 11.4$ units, concentration in the reaction mixture $0.44 \mu \mathrm{g} / \mathrm{mL}$ ) were added. The reaction mixture was incubated at $80{ }^{\circ} \mathrm{C}$ for $24 \mathrm{~h}$ (monitored by HPLC system 1). The solution was neutralized with $2 \mathrm{~N} \mathrm{HCl}$ and concentrated in vacuo to ca. $35 \mathrm{~mL}$. The precipitate was filtered off, and the filtrate was applied to the column (octadecyl-Si 100 polyol $(0.03 \mathrm{~mm}) ; 4 \times 14 \mathrm{~cm})$. The desired product was eluted with $15 \%$ ethanol in water. Yield $36.1 \mathrm{mg}(0.127 \mathrm{mmol} ; 69 \%)$, purity $98.5 \%$ (HPLC system 4$)$.

HRMS (ESI+): $m / z[\mathrm{M}+\mathrm{H}]^{+}$calc. for $\mathrm{C}_{9} \mathrm{H}_{13} \mathrm{~N}_{6} \mathrm{O}_{5}$ 285.0947; found 285.0930; [Base + $\mathrm{H}]^{+}$calc. for $\mathrm{C}_{4} \mathrm{H}_{5} \mathrm{~N}_{6} \mathrm{O}$ 153.0525; found 153.0515.

${ }^{1} \mathrm{H}$ NMR (700 MHz): $\delta=6.98$ (br. s, $\left.2 \mathrm{H}, \mathrm{NH}_{2}\right), 5.87\left(\mathrm{~d}, J=4.8,1 \mathrm{H}, \mathrm{H}-1^{\prime}\right), 5.50(\mathrm{~s}, 1 \mathrm{H}$, OH-2' $), 5.15$ (br. s, $\left.1 \mathrm{H}, \mathrm{OH}-3^{\prime}\right), 4.78\left(\mathrm{~m}, 1 \mathrm{H}, \mathrm{OH}-5^{\prime}\right), 4.70\left(\mathrm{t}, J=4.9,1 \mathrm{H}, \mathrm{H}-2^{\prime}\right), 4.24(\mathrm{t}, J=4.8$, $\left.1 \mathrm{H}, \mathrm{H}-3^{\prime}\right), 3.94\left(\mathrm{~m}, 1 \mathrm{H}, \mathrm{H}-4^{\prime}\right), 3.59$ (dd, $\left.J=4.6,11.9,1 \mathrm{H}, \mathrm{H}-5^{\prime} \mathrm{a}\right), 3.46(\mathrm{dd}, J=5.9,11.7,1 \mathrm{H}$, $\left.\mathrm{H}-5^{\prime} \mathrm{b}\right) .{ }^{13} \mathrm{C}$ NMR $(176 \mathrm{MHz}): \delta=151.73(\mathrm{C}-4), 87.75\left(\mathrm{C}-1^{\prime}\right), 85.28\left(\mathrm{C}-4^{\prime}\right), 72.47\left(\mathrm{C}-2^{\prime}\right), 70.30$ $\left(\mathrm{C}-3^{\prime}\right), 61.62\left(\mathrm{C}-5^{\prime}\right) .{ }^{15} \mathrm{~N}$ NMR $(71 \mathrm{MHz}): \delta=234.90,156.95$. 


\subsubsection{1-( $\beta$-D-Ribofuranosyl)pyrazolo[3,4-d]pyrimidine-4-one (9)}

Allopurinol ( $25 \mathrm{mg}, 0.184 \mathrm{mmol})$, D-ribose $(42.6 \mathrm{mg}, 0.284 \mathrm{mmol}), \mathrm{MnCl}_{2}(44.7 \mathrm{mg}$, $0.355 \mathrm{mmol}), \mathrm{KCl}(529 \mathrm{mg}, 7.1 \mathrm{mmol})$, and $\mathrm{KH}_{2} \mathrm{PO}_{4}(38.6 \mathrm{mg}, 0.284 \mathrm{mmol})$ were dissolved in Tris. $\mathrm{HCl}$ buffer $(20 \mathrm{mM}, 142 \mathrm{~mL}, \mathrm{pH} 8.0)$ under stirring and heating at $50{ }^{\circ} \mathrm{C}$. ATP disodium salt trihydrate $(215 \mathrm{mg}, 0.355 \mathrm{mmol}), E c R K(0.018 \mathrm{mg}$, 5.7 units, concentration in the reaction mixture $0.13 \mu \mathrm{g} / \mathrm{mL}), E c P P M(0.57 \mathrm{mg}, 2.8$ units, concentration in the reaction mixture $4 \mu \mathrm{g} / \mathrm{mL})$, and $E c P N P(0.052 \mathrm{mg}, 11.4$ units, concentration in the reaction mixture $0.37 \mu \mathrm{g} / \mathrm{mL}$ ) were added. The reaction mixture was incubated at $50{ }^{\circ} \mathrm{C}$ for $24 \mathrm{~h}$ (monitored by HPLC system 4). The solution was neutralized with $2-\mathrm{N} \mathrm{HCl}$ and concentrated in vacuo to ca. $35 \mathrm{~mL}$. The precipitate was filtered off, and the filtrate was applied to the column (octadecyl-Si 100 polyol $(0.03 \mathrm{~mm}$ ); $4 \times 14 \mathrm{~cm}$ ). The desired product was eluted with $10 \%$ ethanol in water. Yield $41.6 \mathrm{mg}(0.155 \mathrm{mmol} ; 84 \%), 98.5 \%$ purity (HPLC system 4$)$.

$\mathrm{UV}\left(\mathrm{H}_{2} \mathrm{O}, \mathrm{pH} 7.0\right) \lambda \max , \mathrm{nm}\left(\varepsilon, \mathrm{M}^{-1} \mathrm{~cm}^{-1}\right): 250$ (7600), 206 (27600); $\lambda \mathrm{min}, \mathrm{nm}(\varepsilon$, $\left.\mathrm{M}^{-1} \mathrm{~cm}^{-1}\right): 232(5100)$.

HRMS (ESI+): $m / z$ [M + H] $]^{+}$calc. for $\mathrm{C}_{10} \mathrm{H}_{13} \mathrm{~N}_{4} \mathrm{O}_{5}$ 269.0886; found 269.0849; [Base + $\mathrm{H}]^{+}$calc. for $\mathrm{C}_{5} \mathrm{H}_{5} \mathrm{~N}_{4} \mathrm{O}$ 137.0463; found 137.0444.

${ }^{1} \mathrm{H}$ NMR $(700 \mathrm{MHz}): \delta=12.25(\mathrm{~s}, 1 \mathrm{H}, \mathrm{NH}), 8.14(\mathrm{~s}, 1 \mathrm{H}, \mathrm{H}-3), 8.12(\mathrm{~s}, 1 \mathrm{H}, \mathrm{H}-6), 6.06$ $\left(\mathrm{d}, J=4.53,1 \mathrm{H}, \mathrm{H}-1^{\prime}\right), 4.55\left(\mathrm{dt}, J=4.57 ;<0.5,1 \mathrm{H}, \mathrm{H}-2^{\prime}\right), 4.21\left(\mathrm{t}, J=4.8,1 \mathrm{H}, \mathrm{H}-3^{\prime}\right), 3.91(\mathrm{~m}$, $\left.1 \mathrm{H}, \mathrm{H}-4^{\prime}\right), 3.58\left(\mathrm{dd}, J_{5^{\prime} \mathrm{a}, 4}=4.57 ; J_{5^{\prime} \mathrm{a}, 5^{\prime} \mathrm{b}}=11.65,1 \mathrm{H}, \mathrm{H}-5^{\prime} \mathrm{a}\right), 3.43\left(\mathrm{dd}, J_{5^{\prime} \mathrm{b}, 4}=5.96 ; J_{5^{\prime} \mathrm{b}, 5^{\prime} \mathrm{a}}=\right.$ 11.78, 1 H, H-5'b). ${ }^{13} \mathrm{C}$ NMR (176 MHz): $\delta=157.45$ (C-4), 153.98 (C-7a), 148.86 (C-6), 135.37 (C-3), 106.23 (C-4a), $88.38\left(\mathrm{C}-1^{\prime}\right), 85.25\left(\mathrm{C}-4^{\prime}\right), 73.31\left(\mathrm{C}-2^{\prime}\right), 70.81\left(\mathrm{C}-3^{\prime}\right), 62.27\left(\mathrm{C}-5^{\prime}\right) .{ }^{15} \mathrm{~N}$ NMR (71 MHz): $\delta=302.20(\mathrm{~N}-2), 210.47(\mathrm{~N}-7), 204.80(\mathrm{~N}-1), 173.67(\mathrm{~N}-5)$.

2.8.6. 9-( $\beta$-D-Arabinofuranosyl)-2-chloro-6-methoxypurine (10)

Eighty milligrams $(0.25 \mathrm{mmol})$ of riboside (2) were dissolved in $5 \mathrm{~mL}$ of $10 \mathrm{mM}$ $\mathrm{KH}_{2} \mathrm{PO}_{4}$ (pH 7.0). Fifty microliters of $20 \mathrm{mM} \mathrm{Na}_{2} \mathrm{HAsO}_{4}$ and EcPNP $(0.045 \mathrm{mg}, 10$ units, concentration in the reaction mixture $9 \mu \mathrm{g} / \mathrm{mL}$ ) were added. The solution was heated up to $50{ }^{\circ} \mathrm{C}$ and kept for $3 \mathrm{~h}$ until the complete conversion of riboside (2) to base (3) (monitored by HPLC System $\left.3, R_{\mathrm{t}}(2)=7.6 \mathrm{~min}, R_{\mathrm{t}}(3)=6.0 \mathrm{~min}\right)$. The reaction mixture was cooled down to $4{ }^{\circ} \mathrm{C}$ and kept for $12 \mathrm{~h}$. The precipitate was filtered off, washed with cold $\left(4^{\circ} \mathrm{C}\right)$ $10 \mathrm{mM} \mathrm{KH}_{2} \mathrm{PO}_{4}(\mathrm{pH} 7.0)(2 \times 5 \mathrm{~mL})$, and suspended in $167 \mathrm{~mL}$ of $20 \mathrm{mM}$ Tris. $\mathrm{HCl}$, $\mathrm{pH} 8.0$.

D-Arabinose (1.5 g, $10 \mathrm{mmol}), \mathrm{MnCl}_{2}(52.5 \mathrm{mg}, 0.417 \mathrm{mmol}), \mathrm{KCl}(622 \mathrm{mg}, 8.35 \mathrm{mmol})$, and $\mathrm{KH}_{2} \mathrm{PO}_{4}(44.7 \mathrm{mg}, 0.334 \mathrm{mmol})$ were added under stirring and heating at $50{ }^{\circ} \mathrm{C}$. ATP (253 mg, $0.417 \mathrm{mmol}), E c R K(5.4 \mathrm{mg}, 1670$ units, concentration in the reaction mixture $32 \mu \mathrm{g} / \mathrm{mL})$, EcPPM $(68.2 \mathrm{mg}$, 334 units, concentration in the reaction mixture $408 \mu \mathrm{g} / \mathrm{mL})$, and $E c P N P(13.6 \mathrm{mg}, 3000$ units, concentration in the reaction mixture $82 \mu \mathrm{g} / \mathrm{mL})$ were added, and the reaction mixture was incubated at $50{ }^{\circ} \mathrm{C}$ for $48 \mathrm{~h}$ (monitored by HPLC System 3). The solution was neutralized with 2-N hydrochloric acid and concentrated in vacuo to ca. $25 \mathrm{~mL}$. The precipitate was filtered off. The filtrate was applied to the column (octadecyl-Si 100 polyol $(0.03 \mathrm{~mm}) ; 4 \times 16 \mathrm{~cm}$ ), and the product was eluted with a gradient of acetonitrile in water $(0-50 \%, 1 \mathrm{~L}$, flow rate $6 \mathrm{~mL} / \mathrm{min})$. Yield $43 \mathrm{mg}(0.136 \mathrm{mmol}, 54 \%)$, white amorphous powder, purity $97.0 \%$ (HPLC System $3, R_{\mathrm{t}}=9.4 \mathrm{~min}$ ).

$\mathrm{UV}\left(\mathrm{H}_{2} \mathrm{O}, \mathrm{pH}\right.$ 7.0) $\lambda \max , \mathrm{nm}\left(\varepsilon, \mathrm{M}^{-1} \mathrm{~cm}^{-1}\right): 270(10400)$.

HRMS (ESI+): $m / z[\mathrm{M}+\mathrm{H}]^{+}$calc. for $\mathrm{C}_{11} \mathrm{H}_{14} \mathrm{~N}_{4} \mathrm{O}_{5} \mathrm{Cl} 317.0654 / 319.0624 \mathrm{Cl}^{35} / \mathrm{Cl}^{37}$, found 317.0636/319.0609; [Base $+\mathrm{H}]^{+}$calc. for $\mathrm{C}_{6} \mathrm{H}_{6} \mathrm{~N}_{4} \mathrm{OCl} 185.0230 / 187.0200 \mathrm{Cl}^{35} / \mathrm{Cl}^{37}$, found 185.0221/187.0189.

${ }^{1} \mathrm{H}$ NMR (700 MHz): $\delta=8.48$, (s, $\left.1 \mathrm{H}, \mathrm{H}-8\right), 6.27\left(\mathrm{~d}, J=4.8 \mathrm{~Hz}, 1 \mathrm{H}, \mathrm{H}-\mathrm{1}^{\prime}\right), 5.64$ (br.s., $\left.0.6 \mathrm{H}, 2^{\prime}-\mathrm{OH}\right), 5.57$ (br.s, $\left.1 \mathrm{H}, 3^{\prime}-\mathrm{OH}\right), 5.11$ (br.s, $\left.1 \mathrm{H}, 5^{\prime}-\mathrm{OH}\right), 4.24\left(\mathrm{~m}, 1 \mathrm{H}, \mathrm{H}-2^{\prime}\right), 4.14$ (m, $\left.1 \mathrm{H}, \mathrm{H}-3^{\prime}\right), 4.12\left(\mathrm{~s}, 3 \mathrm{H}, \mathrm{O}-\mathrm{CH}_{3}\right), 3.81\left(\mathrm{~m}, 1 \mathrm{H}, \mathrm{H}-4^{\prime}\right), 3.69$ (m, $\left.1 \mathrm{H}, \mathrm{H}-5^{\prime} \mathrm{a}\right), 3.67$ (m, $\left.1 \mathrm{H}, \mathrm{H}-5^{\prime} \mathrm{b}\right)$. ${ }^{13} \mathrm{C}$ NMR (176 MHz): $\delta=160.63$ (C-6), 152.90 (C-4), 151.23 (C-2), 143.57 (C-8), 119.49 (C-5), $84.12\left(\mathrm{C}-4^{\prime}\right), 84.10\left(\mathrm{C}-1^{\prime}\right), 75.56\left(\mathrm{C}-2^{\prime}\right), 74.23\left(\mathrm{C}-3^{\prime}\right), 60.44\left(\mathrm{C}-5^{\prime}\right), 54.87\left(\mathrm{OCH}_{3}\right) .{ }^{15} \mathrm{~N} \mathrm{NMR}$ (71 MHz): $\delta=239.64(\mathrm{~N}-7), 170.65(\mathrm{~N}-9)$. 


\subsubsection{9-( $\beta$-D-Arabinofuranosyl)-2-fluoro-6-methoxypurine (11)}

Thirty-six milligrams $(0.12 \mathrm{mmol})$ of riboside (6) were dissolved in $10 \mathrm{~mL}$ of $10 \mathrm{mM}$ $\mathrm{KH}_{2} \mathrm{PO}_{4}$ (pH 7.0). Fifty microliters of $20 \mathrm{mM} \mathrm{Na}_{2} \mathrm{HAsO}_{4}$ and EcPNP $(0.068 \mathrm{mg}$, 15 units, concentration in the reaction mixture $6.8 \mu \mathrm{g} / \mathrm{mL}$ ) were added. The solution was heated up to $50{ }^{\circ} \mathrm{C}$ and kept for $3 \mathrm{~h}$ until the complete conversion of riboside (6) to base (7) (monitored by HPLC System 3, RT $(6)=7.9 \mathrm{~min}, \mathrm{RT}(7)=6.2 \mathrm{~min}$ ). The reaction mixture was concentrated twice, cooled down to $4{ }^{\circ} \mathrm{C}$, and kept for $16 \mathrm{~h}$. The supernatant was removed, and the precipitate was washed with cold $\left(4^{\circ} \mathrm{C}\right) 10 \mathrm{mM} \mathrm{KH}_{2} \mathrm{PO}_{4}(\mathrm{pH} 7.0)(2 \times 5 \mathrm{~mL})$ and suspended in $80 \mathrm{~mL}$ of $20 \mathrm{mM}$ Tris. $\mathrm{HCl}, \mathrm{pH}$ 8.0. D-Arabinose (720 mg, $4.8 \mathrm{mmol}$ ), $\mathrm{MnCl}_{2}$ (25.2 mg, $0.2 \mathrm{mmol}), \mathrm{KCl}(298 \mathrm{mg}, 4 \mathrm{mmol})$, and $\mathrm{KH}_{2} \mathrm{PO}_{4}(21.4 \mathrm{mg}, 0.16 \mathrm{mmol})$ were added under stirring and heating at $50{ }^{\circ} \mathrm{C}$. ATP $(121 \mathrm{mg}, 0.2 \mathrm{mmol}), E c R K(2.58 \mathrm{mg}$, 800 units, concentration in the reaction mixture $32 \mu \mathrm{g} / \mathrm{mL}), E c P P M(32.7 \mathrm{mg}, 160$ units, concentration in the reaction mixture $408 \mu \mathrm{g} / \mathrm{mL}$ ), and EcPNP (6.55 mg, 1440 units, concentration in the reaction mixture $82 \mu \mathrm{g} / \mathrm{mL}$ ) were added, and the reaction mixture was incubated at $50{ }^{\circ} \mathrm{C}$ for $48 \mathrm{~h}$ (monitored by HPLC System 3). The solution was neutralized with 2-N hydrochloric acid and concentrated in vacuo to ca. $35 \mathrm{~mL}$. The precipitate was filtered off. The filtrate was applied to the column (octadecyl-Si 100 polyol $(0.03 \mathrm{~mm}) ; 4 \times 16 \mathrm{~cm})$, and the product was eluted with a gradient of acetonitrile in water $(0-50 \%, 1 \mathrm{~L}$, flow rate $6 \mathrm{~mL} / \mathrm{min}$ ). Yield $15 \mathrm{mg}(0.05 \mathrm{mmol}, 42 \%)$, white amorphous powder, purity $99.0 \%$ (HPLC System $\left.3, R_{\mathrm{t}}=7.7 \mathrm{~min}\right)$.

$\mathrm{UV}\left(\mathrm{H}_{2} \mathrm{O}, \mathrm{pH} 7.0\right) \lambda \max , \mathrm{nm}\left(\varepsilon, \mathrm{M}^{-1} \mathrm{~cm}^{-1}\right): 254$ (7200), 244 (6050).

HRMS (ESI+): $m / z$ [M + H] $]^{+}$calc. for $\mathrm{C}_{11} \mathrm{H}_{14} \mathrm{~N}_{4} \mathrm{O}_{5} \mathrm{~F}$ 301.0943; found 301.0927; [Base + $\mathrm{H}]^{+}$calc. for $\mathrm{C}_{6} \mathrm{H}_{6} \mathrm{~N}_{4} \mathrm{OF}$ 169.0520; found 169.0503.

${ }^{1} \mathrm{H}$ NMR $(700 \mathrm{MHz}): \delta=8.46,(\mathrm{~s}, 1 \mathrm{H}, \mathrm{H}-8), 6.22\left(\mathrm{~d}, J=5.4,1 \mathrm{H}, \mathrm{H}-1^{\prime}\right), 5.72$ (br. s., $1 \mathrm{H}$, $\left.\mathrm{OH}-2^{\prime}\right), 5.63$ (br. s, $\left.1 \mathrm{H}, \mathrm{OH}-3^{\prime}\right), 5.11$ (br. s, $\left.1 \mathrm{H}, \mathrm{OH}-5^{\prime}\right), 4.23\left(\mathrm{t}, J=5.2,1 \mathrm{H}, \mathrm{H}-2^{\prime}\right), 4.14(\mathrm{t}$, $\left.J=5.4,1 \mathrm{H}, \mathrm{H}-3^{\prime}\right), 4.12\left(\mathrm{~s}, 3 \mathrm{H}, \mathrm{O}-\mathrm{CH}_{3}\right), 3.80\left(\mathrm{~m}, 1 \mathrm{H}, \mathrm{H}-4^{\prime}\right), 3.70\left(\mathrm{dd}, J=3.7,12.0,1 \mathrm{H}, \mathrm{H}-5^{\prime} \mathrm{a}\right)$, $3.66\left(\mathrm{dd}, J=4.8,12.0,1 \mathrm{H}, \mathrm{H}-5^{\prime} \mathrm{b}\right) .{ }^{13} \mathrm{C}$ NMR $(176 \mathrm{MHz}): \delta=161.98\left(\mathrm{~d}, J_{\mathrm{C} 6, \mathrm{~F}}=17.0 \mathrm{~Hz}, \mathrm{C}-6\right)$, $156.98\left(\mathrm{~d}, J_{\mathrm{C} 2, \mathrm{~F}}=210.8 \mathrm{~Hz}, \mathrm{C}-2\right), 153.00\left(\mathrm{~d}, J_{\mathrm{C} 4, \mathrm{~F}}=18.8 \mathrm{~Hz}, \mathrm{C}-4\right), 143.38(\mathrm{C}-8), 118.64\left(\mathrm{~d}, J_{\mathrm{C} 5, \mathrm{~F}}\right.$ $=4.5 \mathrm{~Hz}, \mathrm{C}-5), 84.09\left(\mathrm{C}-4^{\prime}\right), 84.05\left(\mathrm{C}-1^{\prime}\right), 75.59\left(\mathrm{C}-2^{\prime}\right), 74.18\left(\mathrm{C}-3^{\prime}\right), 60.44\left(\mathrm{C}-5^{\prime}\right), 54.97\left(\mathrm{OCH}_{3}\right)$. ${ }^{15} \mathrm{~N}$ NMR $(71 \mathrm{MГ}): \delta=239.9(\mathrm{~N}-7), 200.11(\mathrm{~N}-3), 170.71(\mathrm{~N}-9)$.

\section{Results}

To create a polyenzymatic cascade for the synthesis of modified nucleosides, it was necessary to obtain a sufficient amount of recombinant ribokinase, phosphopentomutase, and nucleoside phosphorylases. We believe that the enzymes from Escherichia coli and thermophilic bacteria Thermus species 2.9 and Thermus thermophilus HB27 have the best prospects for research and subsequent use. The purifications of all the enzymes (except TthPPM) were obtained earlier, the methods of their isolation were optimized, and the substrate characteristics were studied [21-24,28,29].

To obtain TthPPM, an expression vector was constructed. The TT_RS08405 gene obtained by the amplification of genomic DNA from Thermus thermophilus HB27 was cloned into the pET-23a+ vector. A strain E. coli NiCo21(DE3)/pER-PPM-Tth producing a soluble-form enzyme was created, and a purification protocol was developed. The technique includes the stages of heat precipitation of contaminating proteins and DNA, anion exchange, metal chelate affinity, and size-exclusion chromatography.

Preparations of purified enzymes were characterized by the purity, the content of oligomeric forms, and enzymatic activity (Table 1). 
Table 1. The characteristics of the purified recombinant enzymes.

\begin{tabular}{|c|c|c|c|c|c|}
\hline Name & Enzyme & Gene & Concentration ${ }^{*}, \mathrm{mg} / \mathrm{mL}$ & Oligomer form ** & Purity, $\% * * *$ \\
\hline TspRK & $\begin{array}{c}\text { Ribokinase Thermus } \\
\text { species } 2.9\end{array}$ & QT17_05185 & 13.4 & $\begin{array}{l}63 \mathrm{kD} \\
\text { adimer }\end{array}$ & 94.3 \\
\hline TthPPM & $\begin{array}{l}\text { Phosphopentamutase } \\
\text { Thermus thermophilus HB27 }\end{array}$ & TT_RS08405 & 10.1 & $\begin{array}{c}43 \mathrm{kD} \\
\text { amonomer }\end{array}$ & 70.5 \\
\hline TthPNPI & $\begin{array}{c}\text { Purine nucleoside } \\
\text { phosphorylase I Thermus } \\
\text { thermophilus HB27 }\end{array}$ & TT_RS05405 & 9.8 & $\begin{array}{c}153 \mathrm{kD} \\
\text { ahexamer }\end{array}$ & 96.4 \\
\hline TthPNPII & $\begin{array}{c}\text { Purine nucleoside } \\
\text { phosphorylase II Thermus } \\
\text { thermophilus HB27 }\end{array}$ & TT_RS00985 & 10.3 & $\begin{array}{c}179 \mathrm{kD} \\
\text { ahexamer }\end{array}$ & 95.0 \\
\hline EcRK & Ribokinase E. coli $\mathrm{K} 12$ & AAA51476.1 & 6.9 & $\begin{array}{c}66 \mathrm{kD} \\
\text { adimer }\end{array}$ & 83.2 \\
\hline EcPPM & $\begin{array}{c}\text { Phosphopentamutase } \\
\text { E. coli } \mathrm{K} 12\end{array}$ & AAA97279.1 & 11.0 & $\begin{array}{c}44 \mathrm{kD} \\
\text { amonomer }\end{array}$ & 85.4 \\
\hline$E c \mathrm{PNP}$ & $\begin{array}{c}\text { Purine nucleoside } \\
\text { phosphorylase E. coliK12 }\end{array}$ & AAA24401.1 & 30.1 & $\begin{array}{c}155 \mathrm{kD} \\
\text { ahexamer }\end{array}$ & 95.8 \\
\hline
\end{tabular}

* Analysis by Lowry method. ${ }^{* *}$ Analysis by size exclusion chromatography. ${ }^{* * *}$ Analysis by protein electrophoresis in SDS-PAGE.

\subsection{Study of the Influence of Various Factors on TthPPM Activity}

The effect of $\mathrm{pH}$, reaction mixture temperature, divalent cations, and cofactor (glucose1,6- bisphosphate) on TthPPM activity was studied. The enzymatic activity of TthPPM was calculated, allowing for the content of impurity proteins.

The studied phosphopentomutase showed the maximal activity at $\mathrm{pH} 8.0$ (Figure 1a). The enzyme was active in a wide temperature range; the highest activity was observed in the temperature range from 80 to $90^{\circ} \mathrm{C}$ (Figure 1b).

a)

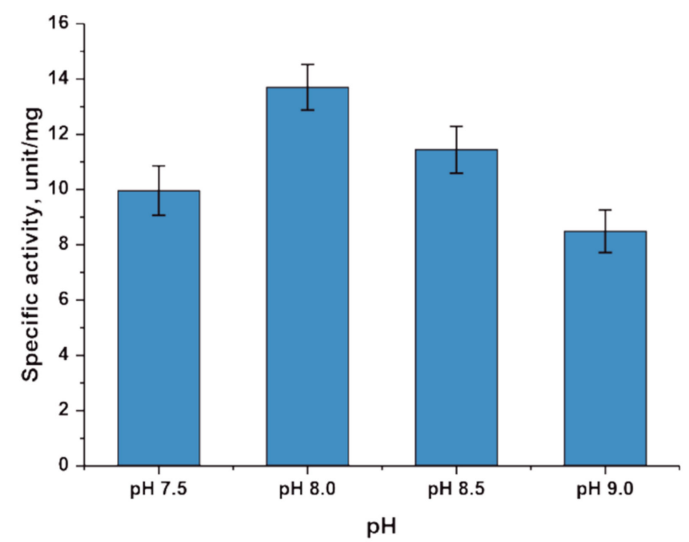

b)

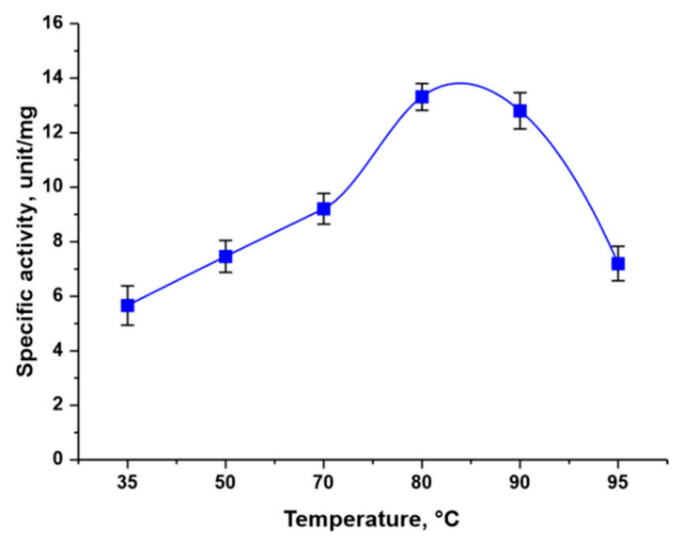

Figure 1. Dependence of TthPPM activity on various factors: (a) $\mathrm{pH}$ and (b) temperature. Reaction conditions: (a) $0.5 \mathrm{mM}$ adenine, $0.5 \mathrm{mM}$ D-ribose 5-phosphate, $0.5 \mathrm{mM} \mathrm{MnCl} 2,20 \mathrm{mM}$ Tris. $\mathrm{HCl}$ ( $\mathrm{pH}$ from 7.5 to 9.0), $0.5 \mu \mathrm{g}$ TthPPM, and $2.7 \mu \mathrm{g}$ TthPNPII in a $0.25 \mathrm{~mL}$ reaction mixture, $80^{\circ} \mathrm{C}$ and (b) $0.5 \mathrm{mM}$ adenine, $0.5 \mathrm{mM}$ D-ribose 5-phosphate, $0.5 \mathrm{mM} \mathrm{MnCl}_{2}$, $20 \mathrm{mM}$ Tris $\cdot \mathrm{HCl}, \mathrm{pH}$ 8.0, $0.1 \mu \mathrm{g}$ TthPPM, and $2.7 \mu \mathrm{g}$ TthPNPII in a $0.25 \mathrm{~mL}$ reaction mixture, temperature 35, 50, 70, 80, 90, and $95{ }^{\circ} \mathrm{C}$.

The measurement of activity in the presence of chlorides of various metals showed that phosphopentomutase can bind various divalent cations, while the maximal activity was observed in the presence of a manganese cation. In the absence of divalent metal cations, the activity of the enzyme significantly decreased (Figure 2a). The studied phosphopentomutase exhibited maximal activity at a concentration of manganese ions of $0.5 \mathrm{mM}$ (Figure 2b). 
a)

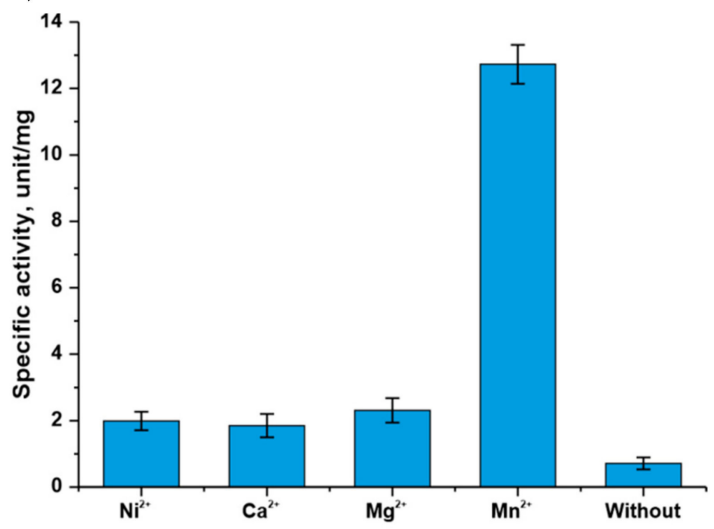

b)

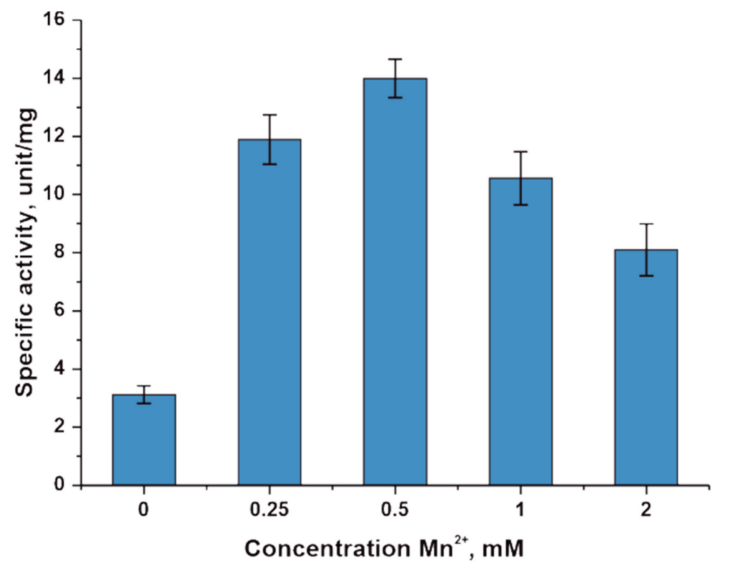

Figure 2. Dependence of TthPPM activity on various factors: (a) divalent cations and (b) $\mathrm{Mn}^{2+}$ concentration. Reaction conditions: (a) $0.5 \mathrm{mM}$ adenine; $0.5 \mathrm{mM}$ D-ribose 5-phosphate; $0.5 \mathrm{mM} \mathrm{MnCl}, \mathrm{MgCl}_{2}, \mathrm{NiCl}_{2}, \mathrm{or} \mathrm{CaCl}_{2}$ and without cation; $20 \mathrm{mM}$ Tris. $\mathrm{HCl}, \mathrm{pH} 8.0 ; 0.1 \mu \mathrm{g}$ TthPPM and $2.7 \mu \mathrm{g}$ TthPNPII in a $0.25 \mathrm{~mL}$ reaction mixture, temperature $80^{\circ} \mathrm{C}$ and $(\mathbf{b})$ $0.5 \mathrm{mM}$ adenine, $0.5 \mathrm{mM}$ D-ribose 5-phosphate, from 0 - to $5 \mathrm{mM} \mathrm{MnCl}_{2}, 20 \mathrm{mM}$ Tris $\cdot \mathrm{HCl}, \mathrm{pH} 8.0,0.1 \mu \mathrm{g}$ TthPPM, and $2.7 \mu \mathrm{g}$ TthPNPII in a $0.25 \mathrm{~mL}$ reaction mixture, temperature $80^{\circ} \mathrm{C}$.

Glucose-1,6-bisphosphate increased the activity of TthPPM (Figure 3), although its presence in the reaction was not necessary.

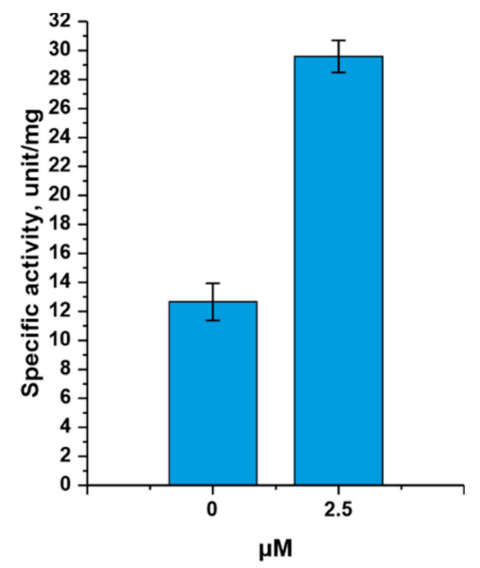

Figure 3. Dependence of TthPPM activity on the cofactor concentration. Reaction conditions: $0.5 \mathrm{mM}$ adenine, $0.5 \mathrm{mM}$ D-ribose 5-phosphate, $0.5 \mathrm{mM} \mathrm{MnCl}_{2}, 20 \mathrm{mM}$ Tris. $\mathrm{HCl}, \mathrm{pH}$ 8.0, 0- or $2.5 \mu \mathrm{M}$ glucose1,6-bisphosphate, $0.2 \mu \mathrm{g}$ TthPPM, and $1.4 \mu \mathrm{g}$ TthPNPII in a $0.25 \mathrm{~mL}$ reaction mixture, temperature $80^{\circ} \mathrm{C}$.

\subsection{Determination of the Kinetic Parameters for TthPPM}

The kinetic parameters were determined with natural substrates: D-ribose-5-phosphate (R5P) and 2-deoxy-D-ribose-5-phosphate (dR5P) (Table 2 and Figure 4).

The data obtained indicate that the affinity of D-ribose-5-phosphate and 2-deoxy-Dribose-5-phosphate for the active site of TthPPM differs insignificantly. Thus, the presence of an $\mathrm{OH}$ group in the second position does not significantly affect the binding to the active site. At the same time, the reaction rate in the active site for D-ribose-5-phosphate is 7.1 times higher.

The activity of all the enzymes used in this study was determined (Table 3). For thermophilic enzymes, the activity was determined at $80^{\circ} \mathrm{C}$ and, for mesophilic enzymes, at $37^{\circ} \mathrm{C}$. As the activity of $T$ spRK was 16 times lower compared to $E c R K$, more proteins were added to the reaction mixture. In this case, the activity of phosphopentomutase and purine nucleoside phosphorylase II (with respect to adenosine) of Thermus thermophilus was 
higher than that of E. coli enzymes, while the activity of TthPNPI (with respect to inosine) and $E c P N P$ was the same.

Table 2. Kinetics of $\alpha$-D-ribose 1-phosphate and 2-deoxy- $\alpha$-D-ribose 1-phosphate synthesis by phosphopentomutase Thermus thermophilus HB27. Each reaction mixture $(100 \mu \mathrm{L}, 20 \mathrm{mM}$ Tris. $\mathrm{HCl}$, $\mathrm{pH}$ 8.0) contained 1-mM adenine, $0.5 \mathrm{mM} \mathrm{MnCl}_{2}$, and $0.25 \mu \mathrm{M} \alpha$-D-glucose 1,6-bisphosphate, from 0.011 to $1.8 \mathrm{mM}$ D-ribose 5-phosphate or 2-deoxy-D-ribose 5-phosphate, 0.02 or $0.05 \mu \mathrm{g}$ TthPPM, and $0.5 \mu \mathrm{g}$ TthPNPII. The reaction mixtures were incubated $2 \mathrm{~min}$ at $80^{\circ} \mathrm{C}$.

\begin{tabular}{ccccc}
\hline Substrate & $\mathbf{K}_{\mathbf{M}}, \mathbf{m M}$ & $\mathbf{V}_{\mathbf{m a x}}, \boldsymbol{\mu \mathbf { m o l }} \mathbf{m} \mathbf{m i n} \cdot \mathbf{m g}$ & $\mathbf{k}_{\mathbf{c a t}}, \mathbf{s}^{-\mathbf{1}}$ & $\mathbf{k}_{\mathbf{c a t}} / \mathbf{K}_{\mathbf{M}}, \mathbf{s}^{-\mathbf{1}} \cdot \mathbf{M}^{-\mathbf{1}}$ \\
\hline $\begin{array}{c}\text { D-ribose } \\
\text { 5-phosphate }\end{array}$ & $0.19 \pm 0.02$ & $3.50 \pm 0.40$ & $2.50 \pm 0.30$ & $1.3 \times 10^{4}$ \\
\hline $\begin{array}{c}\text { 2-deoxy-D-ribose } \\
\text { 5-phosphate }\end{array}$ & $0.25 \pm 0.04$ & $0.48 \pm 0.08$ & $0.35 \pm 0.06$ & $1.4 \times 10^{3}$ \\
\hline
\end{tabular}

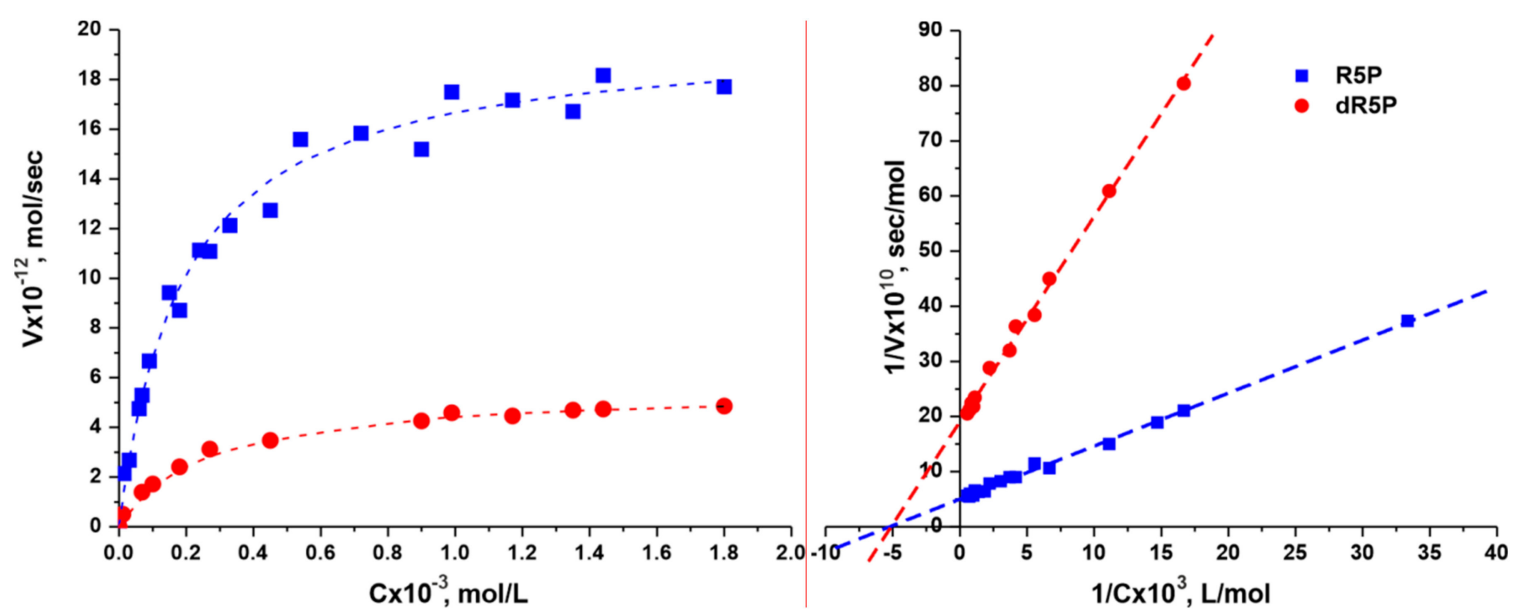

Figure 4. Michaelis-Menten and Lineweaver-Burk plots for $\alpha$-D-ribose 1-phosphate and 2-deoxy- $\alpha$-D-ribose 1-phosphate synthesis by TthPPM.

Table 3. Specific enzymatic activity of the recombinant enzymes.

\begin{tabular}{ccc}
\hline Enzyme & Substrate & Activity, Units/mg \\
\hline TspRK & D-ribose & $19 \pm 3$ \\
\hline \multirow{2}{*}{ TthPPM } & D-ribose 5-phosphate & $14 \pm 3$ \\
\cline { 2 - 3 } & 2-deoxy-D-ribose 5-phosphate & $2.8 \pm 0.7$ \\
\hline TthPNPI & Inosine & $180 \pm 30$ \\
\hline TthPNPII & Adenosine & $200 \pm 30$ \\
\hline$E c R K$ & D-ribose & $310 \pm 40$ \\
\hline$E c$ PPM & D-ribose 5-phosphate & $4.9 \pm 0.9$ \\
\hline \multirow{2}{*}{$E c$ PNP } & Inosine & $220 \pm 30$ \\
\hline
\end{tabular}

\subsection{Synthesis of Modified Heterocyclic Bases for Cascade Synthesis of Nucleosides}

The use of a thermophilic cascade could be advantageous in the synthesis of 2,6disubstituted purine arabinosides, since the solubility of 2-chloro- and 2-fluoradenine (for example) is known to be under $0.4 \mathrm{mM}$ at $50{ }^{\circ} \mathrm{C}$. Model reactions to determine the effectiveness of the thermophilic and mesophilic cascades were performed using 2-chloroadenine as a pentose acceptor. 
After identifying the polyenzymatic cascade conditions, it was useful to synthesize both already known nucleosides (8-azaguanosine and allopurinol riboside) and new modified nucleosides using the cascade approach.

We decided to synthesize two bases: 2-chloro- and 2-fluoro-6-methoxypurine, and to obtain the corresponding arabinosides by a cascade of enzymes. From our experience, the optimal approach to the synthesis of 2-chloro- and 2-fluoro-6-methoxypurines is chemicalenzymatic (Figures 5 and 6 ). This variant of the synthesis has not been previously described in the literature.

All chemical transformations of bases were carried out on ribonucleosides with acetyl protection of the ribose residue. In this case, the protected ribose acts as a protection for the highly reactive N9 in purine.

In addition, the solubility of the nucleosides (both protected and unprotected) in most organic solvents is significantly higher than the solubility of the corresponding bases.

\subsubsection{Synthesis of 2-Chloro-6-methoxypurine (3)}

The scheme of 2-chloro-6-methoxypurine (3) synthesis is shown in Figure 5.

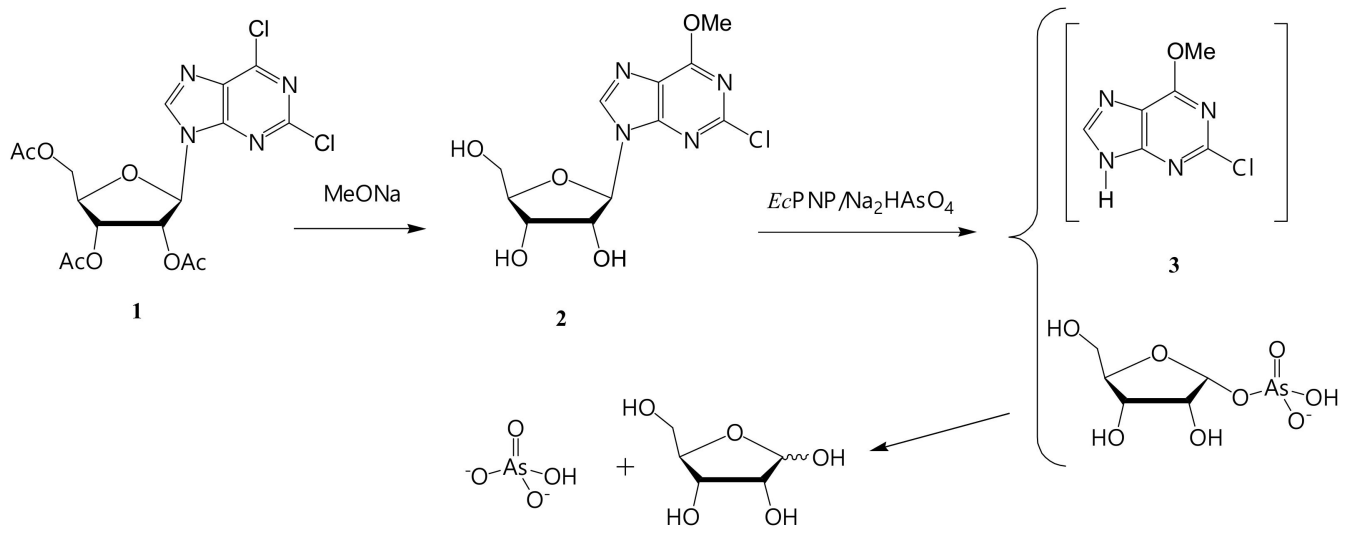

Figure 5. Synthesis of 2-chloro-6-methoxypurine (3).

Riboside (2) was prepared from 9-(2,3,5-tri-O-acetyl-D-ribofuranosyl)-2,6-dichloropurine (1) by a treatment with sodium methylate in methanol, followed by crystallization from the reaction mixture (after neutralization with $\mathrm{HCl}$ ). Additional purification was performed by column chromatography. Riboside (2) was obtained with $44 \%$ yield and 92.9\% purity (HPLC).

Base (3) was obtained from nucleoside (2) using an enzymatic arsenolysis of the glycosidic bond [32].

E. coli PNP can perform arsenolysis of a nucleoside bond. 1- $\alpha$-Ribose arsenate is formed in the active site instead of 1- $\alpha$-phosphate. Ribose arsenate is rapidly hydrolyzed to ribose and inorganic arsenate, and the equilibrium of the enzymatic reaction shifts towards the formation of base (3). E. coli $\mathrm{PNP}$ and $\mathrm{Na}_{2} \mathrm{HAsO}_{4}$ were added to the riboside (2) solution. The reaction mixture was incubated at $60^{\circ} \mathrm{C}$ for several hours. Then, the mixture was concentrated to a minimal volume and kept at $4{ }^{\circ} \mathrm{C}$ for a while. The precipitate (2-chloro-6-methoxypurine (3)) was used in the synthesis of arabinoside without additional purification.

\subsubsection{Synthesis of 2-Fluoro-6-methoxypurine (7)}

The starting compound for the preparation of 2-fluoro-6-methoxypurine (7) was 2amino-6-chloropurine riboside triacetate (4), obtained by the method [27]. The substitution of the 2-amino group with fluorine was performed by the action of $70 \% \mathrm{HF}$ in pyridine (Figure 6).

Product (5) was isolated by column chromatography on silica gel. 2-Fluoro-6-methoxyriboside (6) was prepared by a treatment with sodium methylate in methanol according 
to the procedure described above. The target riboside of 2-fluoro-6-methoxypurine was isolated by column chromatography on a silica gel. The yield of nucleoside (6) was $44 \%$, and the byproduct, according to NMR data, was 2,6-dimethoxypurine riboside. The target product (7) was synthesized by the same method as base (3) and used in the subsequent transformation without purification.

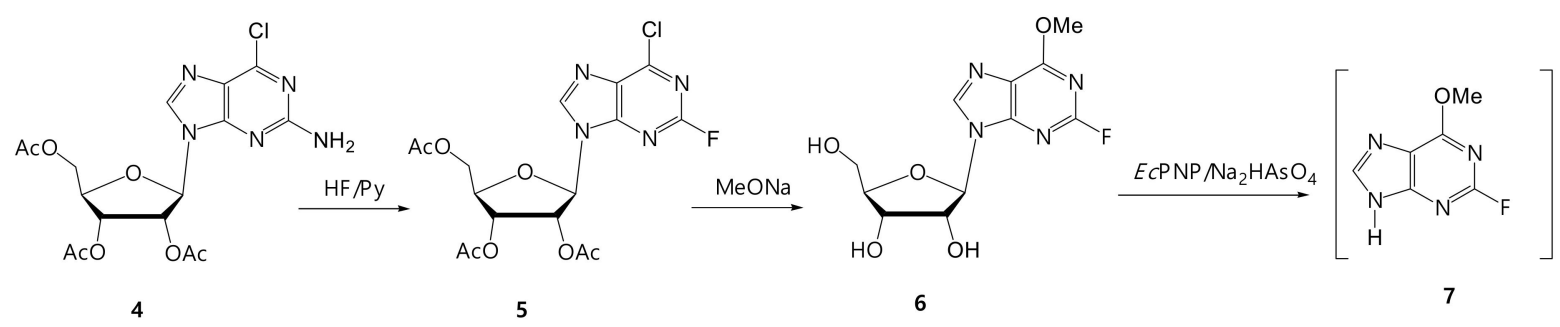

Figure 6. Synthesis of 2-fluoro-6-methoxypurine (7).

\subsection{Conditions for Poly-Enzymatic Cascades Using Mesophilic and Thermophilic Enzymes}

A series of poly-enzymatic cascades using recombinant thermophilic enzymes and E. coli enzymes was performed. The cascade of enzymatic reactions includes the sequential conversion of D-pentoses to 5-monophosphates, catalyzed by ribokinase (RK), the conversion of 5-phosphates to $\alpha$-D-pentose-1-phosphates, catalyzed by phosphopentomutase (PPM), and the condensation of them with the corresponding base, catalyzed by purine nucleoside phosphorylase (PNP). This leads to the formation of the desired $\beta$-D-nucleoside (Figure 7). The reactions were monitored using the liquid chromatography-mass spectrometry (LC-MS) method.
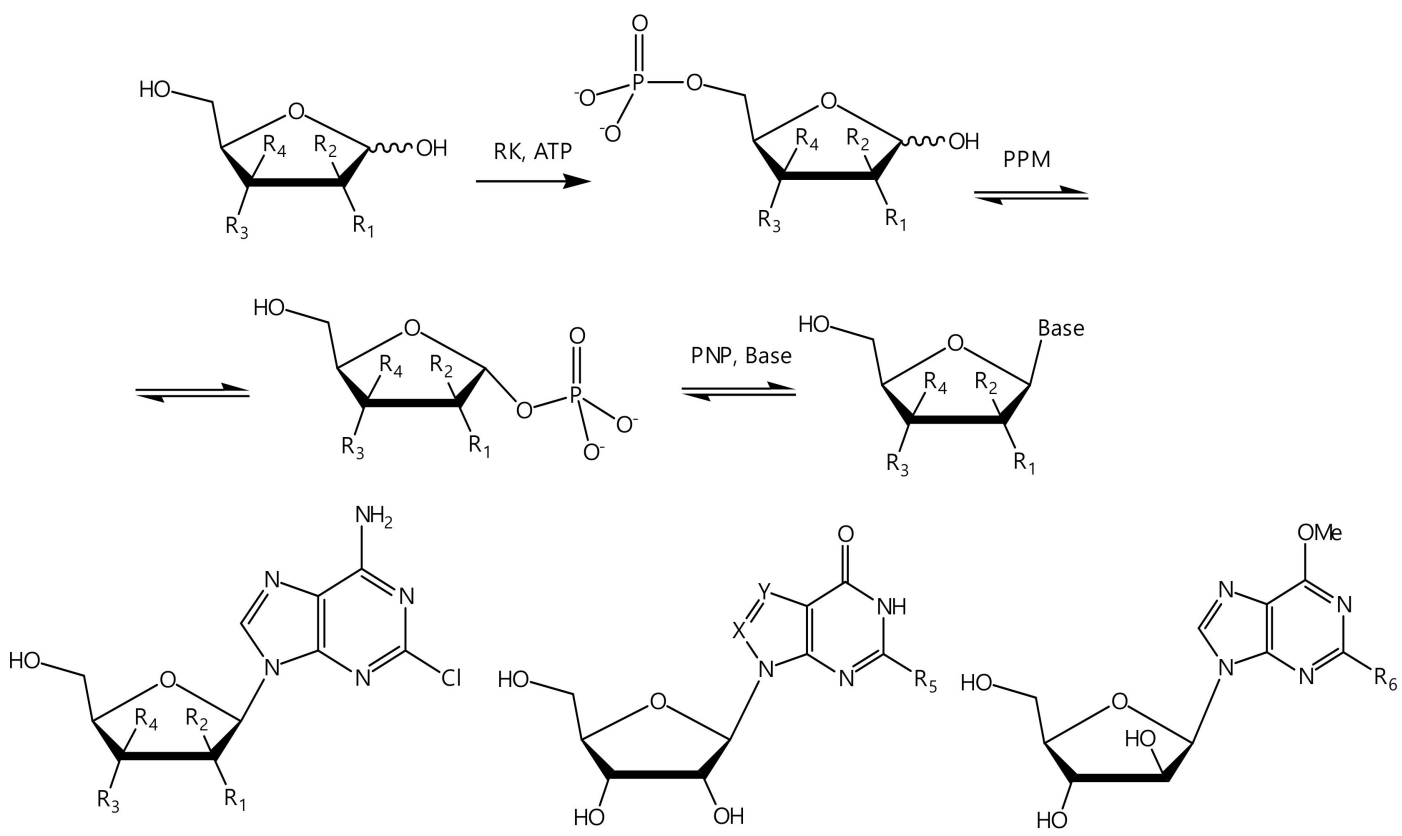

Pentofuranose residue:

$\mathrm{R}_{1}, \mathrm{R}_{3}=\mathrm{OH}, \mathrm{R}_{2}, \mathrm{R}_{4}=\mathrm{H}$ - D-ribose

$\mathrm{R}_{2}, \mathrm{R}_{3}=\mathrm{OH}, \mathrm{R}_{1}, \mathrm{R}_{4}=\mathrm{H}$ - D-arabinose

$X, Y=N, R_{5}=\mathrm{NH}_{2}-8$-azaguanosine, 8 $X=N, Y=C, R_{5}=H$ - allopurinol riboside, 9

$\mathrm{R}_{6}=\mathrm{Cl}$ - 2-chloro-6-methoxy arabinoside, 10 $\mathrm{R}_{2}, \mathrm{R}_{3}=\mathrm{OH}_{1}, \mathrm{R}_{1}, \mathrm{R}_{4}=H$ - D-arabinose
$\mathrm{R}_{1}, \mathrm{R}_{4}=\mathrm{OH}, \mathrm{R}_{2}, \mathrm{R}_{3}=\mathrm{H}$ - D-xylose

$\mathrm{R}_{3}=\mathrm{OH}, \mathrm{R}_{1}, \mathrm{R}_{2}, \mathrm{R}_{4}=\mathrm{H}$ - 2-deoxy-D-ribose

$\mathrm{R}_{3}=\mathrm{OH}, \mathrm{R}_{1}, \mathrm{R}_{2}, \mathrm{R}_{4}=\mathrm{H}-2$-deoxy-D-ribose
$\mathrm{R}_{3}=\mathrm{OH}, \mathrm{R}_{2}=\mathrm{F}_{1}, \mathrm{R}_{1}, \mathrm{R}_{4}=\mathrm{H}$-2-deoxy-2-fluoro-D-arabinose

Figure 7. Synthesis of $\beta$-D-nucleosides from D-pentoses by nucleic and carbohydrate metabolism enzymes.

The conditions for the polyenzymatic cascade with mesophilic E. coli enzymes were determined earlier $[18,33]$ : temperature $50{ }^{\circ} \mathrm{C}, \mathrm{pH}$ 8.0, and the presence of potassium 
chloride and manganese ions. In the case of thermophilic enzymes, the conditions differed only in temperature $\left(80^{\circ} \mathrm{C}\right)$.

The conditions for performing the poly-enzymatic cascade with recombinant thermophilic enzymes with various D-pentoses are presented in Table 4.

Table 4. Conditions for performing the polyenzymatic cascades of nucleoside synthesis with recombinant thermophilic and mesophilic enzymes with various D-pentoses.

\begin{tabular}{|c|c|c|c|c|c|c|c|}
\hline \multirow{2}{*}{ Nucleoside } & \multirow{2}{*}{$\begin{array}{c}\text { 2ClAde } \\
\text { Conc., } \mathrm{mM}\end{array}$} & \multirow{2}{*}{$\begin{array}{l}\text { ATP Conc., } \\
\mathrm{mM}\end{array}$} & \multirow{2}{*}{ D-Pentose Conc. } & \multicolumn{3}{|c|}{ Enzyme Ratio, Units } & \multirow{2}{*}{$\begin{array}{l}\text { Max. Nucleoside } \\
\text { Content, } \%\end{array}$} \\
\hline & & & & RK & PPM & PNP & \\
\hline \multicolumn{8}{|c|}{ Thermus thermophilus } \\
\hline Ribo-2ClAde & \multirow{5}{*}{0.34} & \multirow{5}{*}{2.5} & $\begin{array}{c}2 \mathrm{mM} \\
\text { D-ribose }\end{array}$ & $0.3^{*}$ & 0.1 & $0.5^{* *}$ & 78 (30 min) \\
\hline dRibo-2ClAde & & & $\begin{array}{c}2 \mathrm{mM} \\
\text { 2-deoxy-D-ribose }\end{array}$ & $0.3^{*}$ & 0.1 & $0.5^{* *}$ & $61(30 \mathrm{~min})$ \\
\hline Ara-2ClAde & & & $\begin{array}{c}60 \mathrm{mM} \\
\text { D-arabinose }\end{array}$ & $11 *$ & 1 & $10^{* *}$ & $52(4 \mathrm{~h})$ \\
\hline Xylo-2ClAde & & & $\begin{array}{c}60 \mathrm{mM} \\
\text { D-xylose }\end{array}$ & $11 *$ & 1 & $10^{* *}$ & $4(24 \mathrm{~h})$ \\
\hline Clofarabine & & & $\begin{array}{c}10 \mathrm{mM} \\
\text { 2-deoxy-2-fluoro-D-arabinose }\end{array}$ & $6 *$ & 1 & $10^{* *}$ & $19(24 \mathrm{~h})$ \\
\hline \multicolumn{8}{|c|}{ Escherichia coli } \\
\hline Ribo-2ClAde & \multirow{5}{*}{0.34} & \multirow{5}{*}{2.5} & $\begin{array}{c}2 \mathrm{mM} \\
\text { D-ribose }\end{array}$ & 0.2 & 0.1 & 0.4 & $91(1 \mathrm{~h})$ \\
\hline dRibo-2ClAde & & & $\begin{array}{c}2 \mathrm{mM} \\
\text { 2-deoxy-D-ribose }\end{array}$ & 0.2 & 0.1 & 0.4 & 73 (30 $\mathrm{min})$ \\
\hline Ara-2ClAde & & & $\begin{array}{c}60 \mathrm{mM} \\
\text { D-arabinose }\end{array}$ & 5 & 1 & 9 & $67(1 \mathrm{~h})$ \\
\hline Xylo-2ClAde & & & $\begin{array}{c}60 \mathrm{mM} \\
\text { D-xylose }\end{array}$ & 5 & 1 & 9 & $26(24 \mathrm{~h})$ \\
\hline Clofarabine & & & $\begin{array}{c}10 \mathrm{mM} \\
\text { 2-deoxy-2-fluoro-D-arabinose }\end{array}$ & 6 & 1 & 4 & $46(24 \mathrm{~h})$ \\
\hline
\end{tabular}

* Ribokinase from Thermus species 2.9 (TspRK, gene QT17_05185) [23] was used. ** Purine nucleoside phosphorylase II (TthPNPII) was used.

When selecting conditions for cascade reactions, previous experience with these enzymes was taken into account $[18,22,33]$. High concentrations of arabinose and xylose were due to their poor affinity for the active site of ribokinase. In addition, at ATP concentrations above $3 \mathrm{mM}$, ribokinase inhibition is observed.

As we expected, at first glance, the conditions for conducting polyenzymatic cascades of nucleoside synthesis are very similar, except for the temperature of reaction mixtures. However, there is a significant difference in synthesis efficiency and the stability of products under the conditions of obtaining 2-chloroadenine nucleosides.

\subsection{Synthesis of Nucleosides (8-11)}

We attempted to synthesize ribosides of two bases: 8-azaguanine (8-Aza-Gua) and allopurinol (Allop), using both mesophilic and thermophilic pathways. The conditions for the process are presented in Table 5.

For 8-azaguanine, the efficiency of the thermophilic cascade differed slightly from the mesophilic one. Therefore, we decided to synthesize 8-azaguanosine (8) using thermophilic enzymes, since the increased temperature provided acceptable solubility of the starting heterocycle. E. coli enzymes were used to obtain the allopurinol riboside (9), as they provide higher conversion of the base to riboside ( $96 \%$ per day). 
The products were isolated from the reaction mixtures by column chromatography.

The yields of nucleosides (8) and (9) were $69 \%$ and $84 \%$, respectively.

Table 5. Conditions for performing the poly-enzymatic cascade with recombinant thermophilic and mesophilic enzymes with various heterocyclic bases.

\begin{tabular}{|c|c|c|c|c|c|c|c|}
\hline \multirow{2}{*}{ Nucleoside } & \multirow{2}{*}{$\begin{array}{l}\text { D-Rib Conc., } \\
\text { mM }\end{array}$} & \multirow{2}{*}{$\begin{array}{l}\text { ATP Conc., } \\
\mathrm{mM}\end{array}$} & \multirow{2}{*}{$\begin{array}{c}\text { Base, } \\
0.5 \mathrm{mM}\end{array}$} & \multicolumn{3}{|c|}{ Enzyme Ratio, Units } & \multirow{2}{*}{$\begin{array}{l}\text { Max. Nucleoside } \\
\text { Content, \% (LC MS) }\end{array}$} \\
\hline & & & & RK & PPM & PNP & \\
\hline \multicolumn{8}{|c|}{ Thermus thermophilus } \\
\hline Ribo-8azaGua (8) & \multirow{2}{*}{2} & \multirow{2}{*}{2.5} & 8-Aza-Gua & $0.4^{*}$ & 0.1 & $0.4^{* *}$ & $81(24 \mathrm{~h})$ \\
\hline Ribo-Allop (9) & & & Allop & $0.4^{*}$ & 0.1 & $0.4^{* *}$ & $73(8 \mathrm{~h})$ \\
\hline \multicolumn{8}{|c|}{ Escherichia coli } \\
\hline Ribo-8azaGua (8) & \multirow{2}{*}{2} & \multirow{2}{*}{2.5} & 8-Aza-Gua & 0.2 & 0.1 & 0.4 & $86(24 h)$ \\
\hline Ribo-Allop (9) & & & Allop & 0.2 & 0.1 & 0.4 & $96(24 \mathrm{~h})$ \\
\hline
\end{tabular}

* Ribokinase from Thermus species 2.9 (TspRK, gene QT17_05185) [23] was used. ** Purine nucleoside phosphorylase I (TthPNPI) was used.

The main conditions for the cascade synthesis of nucleosides (8-10) are shown in Table 6.

Table 6. Conditions for the cascade synthesis of nucleosides (8-10).

\begin{tabular}{|c|c|c|c|c|c|c|c|c|}
\hline \multirow{2}{*}{ Comp. } & \multirow{2}{*}{$\begin{array}{l}\text { D-Pentose, } \\
\text { mg (mmol) }\end{array}$} & \multirow{2}{*}{$\begin{array}{c}\text { Base, } \\
\text { mg (mmol) }\end{array}$} & \multirow{2}{*}{$\begin{array}{l}\text { Vol., } \\
\text { mL }\end{array}$} & \multicolumn{3}{|c|}{ Enzymes, U } & \multirow{2}{*}{$\begin{array}{l}\text { Time, } \mathrm{h} \\
\text { /Temp, }{ }^{\circ} \mathrm{C}\end{array}$} & \multirow{2}{*}{$\begin{array}{c}\text { Isolated Yield } \\
\text { mg (\%) }\end{array}$} \\
\hline & & & & RK & PPM & PNP & & \\
\hline \multicolumn{9}{|c|}{ Thermus thermophilus } \\
\hline 8 & $\begin{array}{l}\text { Ribose } 42.6 \\
\quad(0.284)\end{array}$ & $\begin{array}{c}\text { 8-Aza-Gua } \\
28(0.184)\end{array}$ & 142 & 11.4 * & 2.8 & $11.4^{* *}$ & $\begin{array}{c}24 / \\
80\end{array}$ & $36.1(69)$ \\
\hline \multicolumn{9}{|c|}{ Escherichia coli } \\
\hline 9 & $\begin{array}{c}\text { Ribose } 42.6 \\
\quad(0.284)\end{array}$ & $\begin{array}{c}\text { Allop } \\
25(0.184)\end{array}$ & 142 & 5.7 & 2.8 & 11.4 & $\begin{array}{c}24 / \\
50\end{array}$ & $41.6(84)$ \\
\hline 10 & $\begin{array}{c}\text { Arabinose } \\
1500 \\
(10)\end{array}$ & $\begin{array}{c}(2) \\
80(0.25)\end{array}$ & 167 & 1670 & 334 & 3000 & $\begin{array}{c}48 / \\
50\end{array}$ & $43.0\left(54^{* * *}\right)$ \\
\hline 11 & $\begin{array}{c}\text { Arabinose } \\
720 \\
(4.8)\end{array}$ & $\begin{array}{c}(6) \\
36(0.12)\end{array}$ & 80 & 800 & 160 & 1440 & $\begin{array}{c}48 / \\
50\end{array}$ & $14.7\left(41^{* * *}\right)$ \\
\hline
\end{tabular}

* Ribokinase from Thermus species 2.9 (TspRK, gene QT17_05185) [23] was used. ** Purine nucleoside phosphorylase I (TthPNPI) was used.

${ }_{* * *}$ Recalculated for the starting riboside.

The synthesis of 9-( $\beta$-D-Arabinofuranosyl)-2-chloro-6-methoxypurine (10) and 9-( $\beta$ D-Arabinofuranosyl)-2-fluoro-6-methoxy-purine (11) was performed without preliminary optimization of the conditions. Only the quantity of E. coli enzymes was doubled. This allowed achieving the conversion of the base into arabinoside for the 2-chloro derivative (10) of $65 \%$ and for the fluorinated analog (11) of $90 \%$ in two days.

The products were isolated by column chromatography. The yields of nucleosides (10) and (11) were $54 \%$ and $42 \%$, respectively.

\section{Discussion}

To understand the peculiarities of the mesophilic and thermophilic cascades in the synthesis of 2-chloroadenine riboside, 2-deoxyriboside, arabinoside, 2-deoxy-2-fluoroD-arabinoside, and xyloside, it is necessary to scrutinize the dynamics of nucleoside formation. 


\subsection{Possibilities of Using Various D-Pentoses in the Synthesis of 2-Chloroadenine Nucleosides}

D-ribose, 2-deoxy-D-ribose, and their phosphates are natural substrates of nucleic acid and carbohydrate metabolism enzymes. Therefore, their conversion in a cascade with thermophilic enzymes proceeded quite intensively. The maximal content of 2-chloroadenosine (Ribo-2ClAde) was 78\% after $30 \mathrm{~min}$, and 2'-deoxy-2-chloroadenosine (dRibo-2ClAde) was $60 \%$ after $30 \mathrm{~min}$ (Figure 8 ). Interestingly, the equilibrium of the cascade synthesis in the synthesis of the 2-chloroadenine riboside by E. coli enzymes was shifted towards nucleoside formation. However, under the conditions of a thermophilic cascade, the maximal product concentration was observed after $30 \mathrm{~min}$, and then, the equilibrium began to shift in the opposite direction, which led to a twofold decrease of product content in the reaction mixture. These results may be due to the increasing hydrolysis rate of $\alpha$-D-ribose-1-phosphate with the temperature increasing [34].

a)

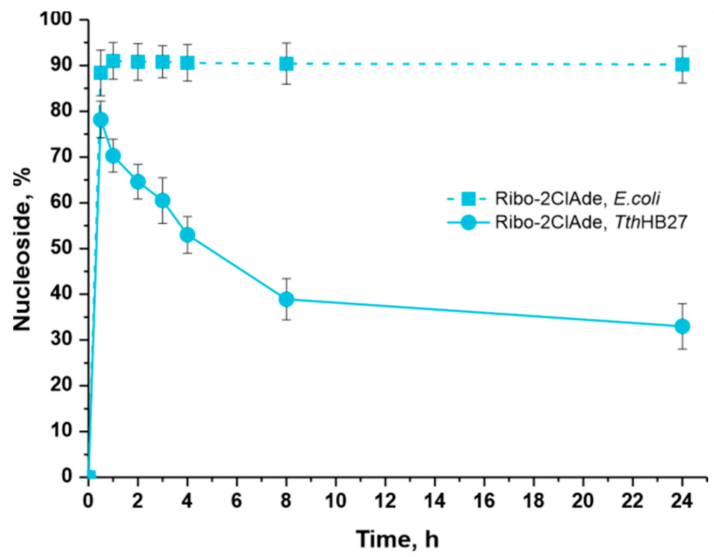

b)

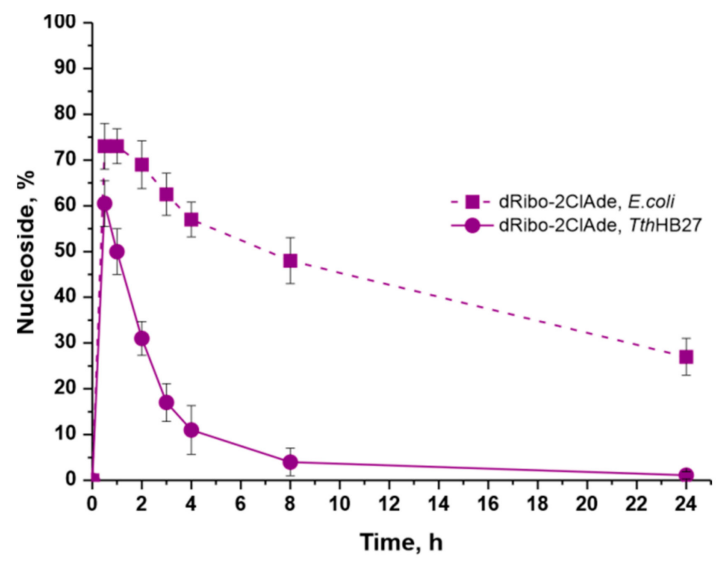

Figure 8. Accumulation of 2-chloroadenosine (a) and 2'-deoxy-2-chloroadenosine (Cladribine) (b) during cascades of enzymatic reactions with thermophilic and mesophilic enzymes (HPLC data).

The content of 2'-deoxy-2-chloroadenosine also sharply decreased over time due to the equilibrium shift towards the opposite reaction because of the hydrolysis of 2-deoxy- $\alpha$ D-ribose-1-phosphate (Figure 8b).

In the cascade reaction with E. coli enzymes, the maximal content of 2-chloroadenosine $(91 \%)$ was observed after one hour and 2 -deoxy-2-chloroadenosine $(73 \%)$ after $30 \mathrm{~min}$. The content of 2-chloroadenosine in the reaction mixture remained constant. The content of 2'-deoxy-2-chloroadenosine gradually decreased over time due to the shift of equilibrium towards the opposite reaction. However, the rate of the opposite reaction was noticeably lower than in the cascade with thermophilic enzymes.

The conversion of D-xylose in the thermophilic cascade was very slow, and the maximal conversion of 2-chloroadenine to 9-( $\beta$-D-xylofuranosyl)-2-chloroadenine was only $3.9 \%$ after $24 \mathrm{~h}$ (Figure $9 \mathrm{a})$. The maximal content of 9-( $\beta$-D-xylofuranosyl)-2-chloroadenine was $26 \%$ after $24 \mathrm{~h}$ in the mesophilic cascade, which is much higher than in the cascade with thermophilic enzymes.

The formation of 9-( $\beta$-D-arabinofuranosyl)-2-chloroadenine from $\mathrm{D}$-arabinose proceeded much more slowly (Figure 9a) compared to the formation of 2-chloroadenosine and $2^{\prime}$-deoxy-2-chloroadenosine (arabinoside content $48.1 \%$ after $24 \mathrm{~h}$ ). The maximal content of 9-( $\beta$-D-arabinofuranosyl)-2-chloroadenine was higher in the mesophilic cascade than in the cascade with thermophilic enzymes (67\% after an hour), but then, its content gradually decreased.

The conversion of 2-deoxy-2-fluoro-D-arabinose to 9-(2'-deoxy-2'-fluoro- $\beta$-D-Arabinofuranosyl)-2-chloroadenine (clofarabine) (Figure 9b) proceeded slower than the synthesis of arabinosides (the maximal content was 19\% after $24 \mathrm{~h}$, from a thermophilic cascade). The conversion of 2-deoxy-2-fluoro-D-arabinose to clofarabine proceeded much better in the mesophilic cascade than in the cascade with thermophilic enzymes (the maximal content of clofarabine was $46 \%$ versus $19 \%$ after $24 \mathrm{~h}$ ). 
a)

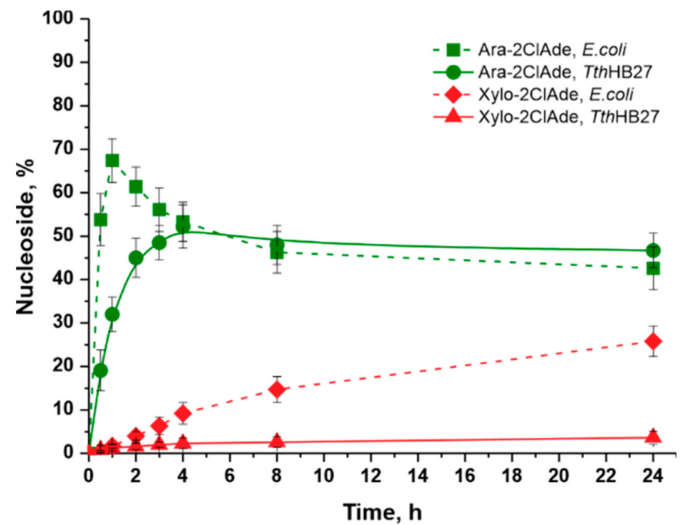

b)

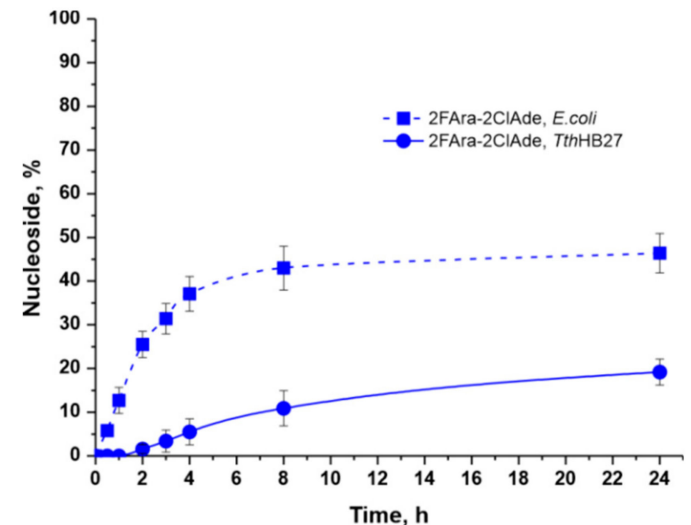

Figure 9. Accumulation of 2-chloroadenine arabinoside and xyloside (a) and 2-chloroadenine 2'-deoxy-2'-fluoro-Darabinoside (Clofarabine) (b) during the cascades of enzymatic reactions with thermophilic and mesophilic enzymes.

Kamel with coworkers [35] investigated the stability of some pentose-1-phosphates at various temperatures. The stability of $\alpha$-D-arabinose-1-phosphate was higher than that of $\alpha$ D-ribose-1-phosphate and 2-deoxy- $\alpha$-D-ribose-1-phosphate, but all these compounds were hydrolyzed. The stability of 2-deoxy-2-fluoro- $\alpha$-D-arabinose-1-phosphate was much higher than that of all other pentose-1-phosphates. A slow decreasing of the product content after $24 \mathrm{~h}$ in the cascade with D-arabinose and the absence of decreasing in the cascade with 2-deoxy-2-fluoro-D-arabinose may be due to a low rate of 1-phosphate intermediate hydrolysis.

The lower rate of D-arabinosides synthesis in comparison with D-ribosides and 2deoxy-D-ribosides can be explained by the different conformations of carbohydrates in the active site of purine nucleoside phosphorylase. For this reason, the rate of D-arabinosides phosphorolysis is also much lower [36].

\subsection{Non-Natural Heterocyclic Bases in the Cascade Synthesis of Nucleosides}

To determine the possibility of practical use of thermophilic or mesophilic cascades, we tried to synthesize several non-natural nucleosides. We selected 8-azaguanine, allopurinol, 2-chloro-6-methoxypurine (3), and 2-fluoro-6-methoxypurine (7) as the heterocyclic bases, taking into account the prospect of subsequent biological testing of the corresponding modified nucleosides.

Synthesis of 8-Azaguanine and Allopurinol Ribosides

We investigated the formation of 9-( $\beta$-D-ribofuranosyl)-8-azaguanine and 9-( $\beta$-Dribofuranosyl) allopurinol from 8 -azaguanine and allopurinol, respectively. In both cases, the transformation proceeded at a high rate. The formation of 9-( $\beta$-D-ribofuranosyl) allopurinol (Figure 10a) in the reaction with $E$. coli enzymes proceeded at a high rate; its maximal content was $95 \%$ after eight hours and did not change over time. The maximal content of 9-( $\beta$-D-ribofuranosyl) allopurinol in the thermophilic cascade was $73 \%$ after eight hours; then, the content decreased. The rate of conversion of 8-azaguanine to 8azaguanosine (8-azaGuo) in the mesophilic cascade was higher than in the cascade with thermophilic enzymes (Figure 10b). 8-Azaguanosine gradually accumulated in the reaction mixture with E. coli enzymes, and its maximum content was $89 \%$ after $24 \mathrm{~h}$.

Using the parameters of the test cascade syntheses, four nucleosides were prepared and isolated. 8-Azaguanosine was synthesized by a thermophilic cascade with a $69 \%$ yield. Allopurinol riboside was obtained by a mesophilic cascade with an $84 \%$ yield. For the first time, 2-chloro-6-methoxypurine (3) and 2-fluoro-6-methoxypurine (7) D-arabinosides were synthesized using a mesophilic cascade (yields $54 \%$ and $41 \%$, respectively).

All synthesized nucleosides were characterized by LC-MS data. The structure was confirmed by NMR spectra: ${ }^{1} \mathrm{H},{ }^{1} \mathrm{H}-{ }^{1} \mathrm{H}-\mathrm{COSY},{ }^{1} \mathrm{H}-{ }^{13} \mathrm{C}-\mathrm{HSQC},{ }^{1} \mathrm{H}-{ }^{13} \mathrm{C}-\mathrm{HMBC},{ }^{1} \mathrm{H}-{ }^{15} \mathrm{~N}-$ $\mathrm{HSQC}$, and ${ }^{1} \mathrm{H}-{ }^{15} \mathrm{~N}-\mathrm{HMBC}$. 
a)

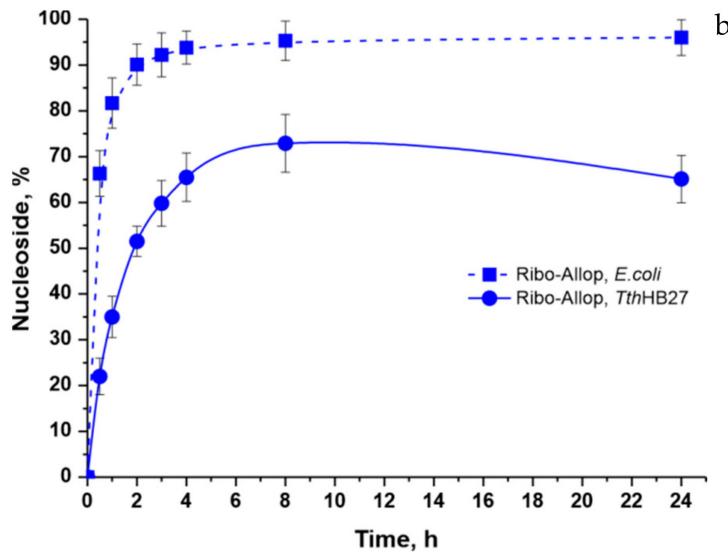

b)

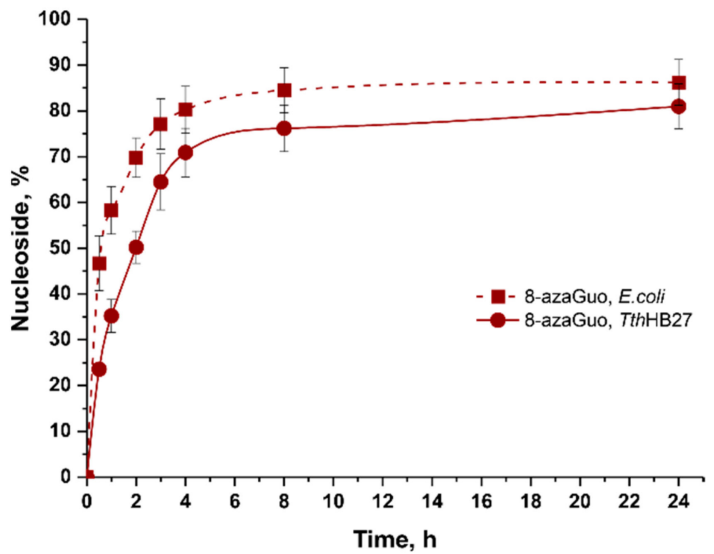

Figure 10. Comparative efficiency of allopurinol (a) and 8-azaguanine (b) ribosides synthesis in mesophilic and thermophilic cascades

The data obtained indicated that, despite the relatively small difference in temperatures when performing the cascade reactions $\left(50\right.$ and $80^{\circ} \mathrm{C}$ ), the rate of product formation in the reactions with $E$. coli enzymes was much higher. The E. coli enzymes also provided a higher content of target products in the reaction mixture. Therefore, they were more appropriate for use in the polyenzymatic synthesis of modified nucleosides.

\section{Conclusions}

To carry out a comparative study of the possibilities of using the RK $\rightarrow$ PPM $\rightarrow$ NP cascade in the synthesis of modified nucleosides, sufficient quantities of genetically engineered enzymes of both E. coli and thermophilic Thermus species 2.9 and Thermus thermophilus HB27 were obtained. Recombinant phosphopenthomutase PPM from Thermus thermophilus HB27 was obtained for the first time: a strain-producer of the soluble form of the enzyme was created, and a procedure for its isolation and chromatographic purification was developed. The influence of the $\mathrm{pH}$, temperature, presence of divalent cations, and cofactor (glucose-1,6-bisphosphate) on the activity of TthPPM was studied. The maximum activity was observed at $\mathrm{pH}$ 8.0. The enzyme is active over a wide temperature range, with the maximum activity in the interval between 80 and $90{ }^{\circ} \mathrm{C}$. Glucose-1,6-bisphosphate significantly increased the activity of $T$ thPPM, although its presence in the reaction was not necessary.

The preparations of all purified enzymes were characterized by purity, the content of the oligomeric forms, and enzymatic activity.

It was shown that the cascade synthesis of the modified nucleosides from D-pentoses could be performed by both the mesophilic and thermophilic enzymes. The following D-pentoses were tested: ribose, 2-deoxyribose, arabinose, xylose, and 2-deoxy-2fluoroarabinose. The efficiency of 2-chloroadenine nucleoside synthesis with mesophilic enzymes decreased in the following order: Rib (92), dRib (74), Ara (66), F-Ara (8), and Xyl (2\%) in $30 \mathrm{~min}$. For thermophilic enzymes, the order was Rib (76), dRib (62), Ara (32), F-Ara (<1), and Xyl (2\%) in $30 \mathrm{~min}$. After one day of incubation, the amounts of 2-chloroadenine riboside (thermophilic cascade), 2-deoxyribosides (both cascades), and arabinoside (mesophilic cascade) decreased roughly by half. Meanwhile, the conversion of the base to 2-fluoroarabinosides and xylosides continued to grow in both cases and reached $20-40 \%$. That was quite acceptable for the preparative synthesis of nucleosides.

Four D-ribose and D-arabinose nucleosides were synthesized on a large scale by a cascade of enzymes. 8-Azaguanine riboside was synthesized by the thermophilic cascade with a $69 \%$ yield. Allopurinol riboside was obtained by the mesophilic cascade with an $84 \%$ yield. For the first time, 2-chloro-6-methoxypurine and 2-fluoro-6-methoxypurine D-arabinosides were synthesized using the mesophilic cascade (yields $54 \%$ and $42 \%$, respectively). The data obtained indicated that, despite the relatively small difference 
in temperatures when performing the cascade reactions $\left(50\right.$ and $\left.80{ }^{\circ} \mathrm{C}\right)$, the rate of the product formation in the reactions with E. coli enzymes was much higher. The E. coli enzymes also provided a higher content of products in the reaction mixtures. Therefore, they are more appropriate for use in the polyenzymatic synthesis of modified nucleosides. The use of thermophilic enzymes and a high reaction temperature might be preferable with low-soluble heterocyclic bases (2-chloroadenine and 2-fluoroadenine), as well as in the case of using carbohydrates that form 1-phosphates with high temperature stability (D-arabinose and 2-deoxy-2-fluoro-D-arabinose).

Supplementary Materials: The following are available online at https:/ / www.mdpi.com/article/10 .3390/biom11040586/s1: Figure S1: Synthesis of 2-chloro-6-methoxypurine (3), Figure S2: The ${ }^{1} \mathrm{H}$ NMR spectrum of compound 1, Figure S3: The ${ }^{1} \mathrm{H}$ NMR spectrum of compound 2, Figure S4: The ${ }^{13} \mathrm{C}$ NMR spectrum of compound 2, Figure S5: The fragment of ${ }^{1} \mathrm{H}-{ }^{15} \mathrm{~N}-\mathrm{HMBC}$ NMR spectrum of nucleoside 2, Figure S6: Synthesis of 2-fluoro-6-methoxypurine (7), Figure S7: The ${ }^{1} \mathrm{H}$ NMR spectrum of compound 4, Figure S8: The ${ }^{1} \mathrm{H}$ NMR spectrum of compound 5, Figure S9: The ${ }^{13} \mathrm{C}$ NMR spectrum of compound 5, Figure S10: The fragment of ${ }^{1} \mathrm{H}-{ }^{15} \mathrm{~N}-\mathrm{HMBC}$ NMR spectrum of nucleoside 5, Figure S11: The ${ }^{1} \mathrm{H}$ NMR spectrum of compound 6, Figure S12: The ${ }^{13} \mathrm{C}$ NMR spectrum of compound 6, Figure S13: The fragment of ${ }^{1} \mathrm{H}-{ }^{15} \mathrm{~N}-\mathrm{HMBC}$ NMR spectrum of nucleoside 6, Figure S14: The ${ }^{1} \mathrm{H}$ NMR spectrum of compound 8, Figure S15: The fragment of ${ }^{1} \mathrm{H}_{-}{ }^{13} \mathrm{C}-\mathrm{HSQC}$ NMR spectrum of nucleoside 8, Figure S16: The fragment of ${ }^{1} \mathrm{H}_{-}{ }^{15} \mathrm{~N}-\mathrm{HMBC}$ NMR spectrum of nucleoside 8, Figure S17: The ${ }^{1} \mathrm{H}$ NMR spectrum of compound 9, Figure S18: The ${ }^{13} \mathrm{C}$ NMR spectrum of compound 9, Figure S19: The fragment of ${ }^{1} \mathrm{H}_{-}{ }^{15} \mathrm{~N}-\mathrm{HMBC}$ NMR spectrum of nucleoside 9, Figure S20: The ${ }^{1} \mathrm{H}$ NMR spectrum of compound 10, Figure S21: The ${ }^{13} \mathrm{C}$ NMR spectrum of compound 10, Figure S22: The fragment of ${ }^{1} \mathrm{H}_{-}{ }^{15} \mathrm{~N}-\mathrm{HMBC}$ NMR spectrum of nucleoside 10, Figure S23: The ${ }^{1} \mathrm{H}$ NMR spectrum of compound 11, Figure S24: The ${ }^{13} \mathrm{C}$ NMR spectrum of compound 11, Figure S25: The fragment of ${ }^{1} \mathrm{H}-{ }^{15} \mathrm{~N}-\mathrm{HMBC}$ NMR spectrum of nucleoside 11.

Author Contributions: Conceptualization, I.V.F., I.D.K., and R.S.E.; methodology, I.V.F., M.A.K., B.Z.E., M.Y.B. and A.L.K.; software, I.V.F. and M.A.K.; validation, B.Z.E., I.V.F., and M.A.K.; investigation, I.V.F., M.A.K., Y.A.A., B.Z.E., M.Y.B., O.O.M., D.D.L., E.A.Z., E.V.D., and A.S.P.; resources, I.V.F. and M.A.K.; data curation, I.D.K. and R.S.E.; writing-original draft preparation, I.V.F., M.A.K., B.Z.E., and I.D.K.; writing-review and editing, I.V.F., I.D.K., A.L.K.; visualization, I.V.F. and M.A.K.; supervision, I.D.K. and R.S.E.; and project administration, I.D.K. and R.S.E. All authors have read and agreed to the published version of the manuscript.

Funding: This research received no external funding.

Institutional Review Board Statement: Not applicable.

Informed Consent Statement: Not applicable.

Data Availability Statement: The data presented in this study are available on request from the corresponding author.

Conflicts of Interest: The authors declare no conflict of interest.

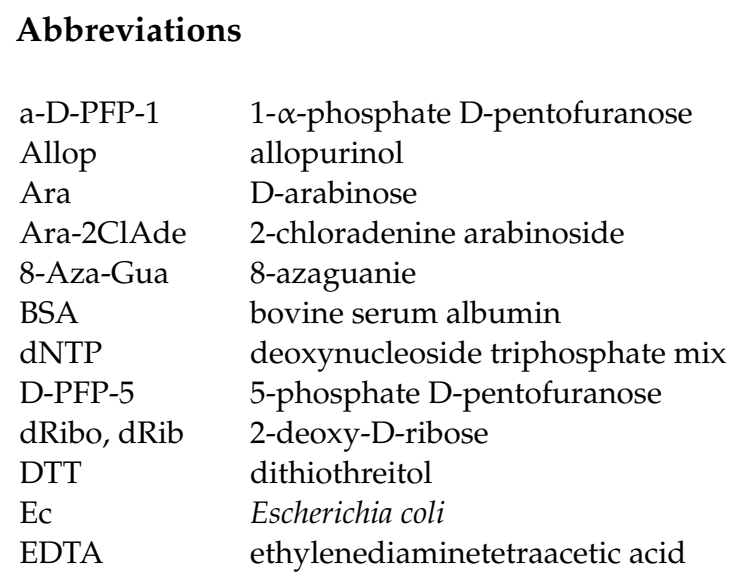




$\begin{array}{ll}\text { F-Ara } & \text { 2-deoxy-2-fluoro-D-arabinose } \\ \text { HMBC } & \text { heteronuclear multiple bond correlation spectroscopy } \\ \text { HSQC } & \text { heteronuclear single quantum correlation spectroscopy } \\ \text { kDa } & \text { kilodalton } \\ \text { IPTG } & \text { isopropyl } \beta \text {-D-1-thiogalactopyranoside } \\ \text { NP } & \text { nucleoside phosphorylase } \\ \text { PMSF } & \text { phenylmethylsulphonyl fluoride } \\ \text { PNP } & \text { purine nucleoside phosphorylase } \\ \text { PPM } & \text { phosphopentomutase } \\ \text { PyNP } & \text { pyrimidine nucleoside phosphorylase } \\ \text { Ribo, Rib } & \text { D-ribose } \\ \text { RK } & \text { ribokinase } \\ \text { TEMED } & \text { tetramethylethylenediamine } \\ \text { TP } & \text { thymidine phosphorylase } \\ \text { Tsp } & \text { Thermus species 2.9 } \\ \text { Tth } & \text { Thermus thermophilus } \\ \text { UP } & \text { uridine phosphorylase } \\ \text { Xyl } & \text { D-xylose } \\ \text { Xylo-2ClAde } & \text { 2-chloroadenine xyloside }\end{array}$

\section{References}

1. Fernández-Lucas, J.; Fresco-Taboada, A.; Acebal, C.; de la Mata, I.; Arroyo, M. Enzymatic synthesis of nucleoside analogues using immobilized 2'-deoxyribosyltransferase from Lactobacillus reuteri. Appl. Microbiol. Biotechol. 2011, 91, 317-327. [CrossRef]

2. Boryski, J. Reactions of transglycosylation in the nucleoside chemistry. Curr. Org. Chem. 2008, 12, 309-325. [CrossRef]

3. Fernández-Lucas, J. Multienzymatic synthesis of nucleic acid derivatives: A general perspective. Appl. Microbiol. Biotechol. 2015, 99, 4615-4627. [CrossRef] [PubMed]

4. Del Arco, J.; Fernández-Lucas, J. Purine and pyrimidine salvage pathway in thermophiles: A valuable source of biocatalysts for the industrial production of nucleic acid derivatives. Appl. Microbiol. Biotechnol. 2018, 102, 7805-7820. [CrossRef] [PubMed]

5. Ding, Q. Synthesis of nucleic acid derivatives by multi-enzymatic systems. In Enzymatic and Chemical Synthesis of Nucleic Acid Derivatives; Fernandez-Lucas, J., Camarasa Rius, M.J., Eds.; Wiley-VCH: Weinheim, Germany, 2019; Volume 6, pp. $129-157$.

6. Lewkowicz, E.S.; Iribarren, A.M. Nucleoside phosphorylases. Cur. Org. Chem. 2006, 10, 1197-1215. [CrossRef]

7. Michailidou, F.; Burnett, D.; Sharma, S.V.; Van Lanen, S.G.; Goss, R.J.M. Natural products incorporating pyrimidine nucleosides. Compr. Nat. Prod. III Third Ed. 2020, 2, 500-536.

8. Michailidou, F.; Chung, C.; Brown, M.J.B.; Bent, A.F.; Naismith, J.H.; Leavens, W.J.; Lynn, S.M.; Sharma, S.V.; Goss, R.J.M. Pac13 is a small, monomeric dehydratase that mediates the formation of the $3^{\prime}$-deoxy nucleoside of pacidamycins. Angew. Chem. Int. Ed. 2017, 56, 12492-12497. [CrossRef] [PubMed]

9. Mikhailopulo, I.A.; Miroshnikov, A.I. Biologically important nucleosides: Modern trends in biotechnology and application. Mendeleev Commun. 2011, 21, 57-68. [CrossRef]

10. Lapponi, M.J.; Rivero, C.W.; Zinni, M.A.; Britos, C.N.; Trelles, J.A. New developments in nucleoside analogues biosynthesis: A review. J. Mol. Catal. B Enzym. 2016, 133, 218-233. [CrossRef]

11. De Benedetti, E.C.; Rivero, C.W.; Britos, C.N.; Lozano, M.E.; Trelles, J.A. Biotransformation of 2,6-diaminopurine nucleosides by immobilized Geobacillus stearothermophilus. Biotechnol. Progr. 2012, 28, 1251-1256. [CrossRef]

12. Almendros, M.; Berenguer, J.; Sinisterra, J.-V. Thermus thermophilus Nucleoside phosphorylases active in the synthesis of nucleoside analogues. Appl. Environ. Microbiol. 2012, 78, 3128-3135. [CrossRef]

13. Kaspar, F.; Neubauer, P.; Kurreck, A. The peculiar case of the hyperthermostable pyrimidine nucleoside phosphorylase from Thermus thermophilus. Chembiochem A Eur. J. Chem. Biol. 2021, 22, 1-7. [CrossRef]

14. Hori, N.; Watanabe, M.; Sunagawa, K.; Uehara, K.; Mikami, Y. Production of 5-methyluridine by immobilized thermostable purine nucleoside phosphorylase and pyrimidine nucleoside phosphorylase from Bacillus stearothermophilus JTS 85. J. Biotechnol. 1991, 17, 121-131. [CrossRef]

15. Taran, S.A.; Verevkina, K.N.; Feofanov, S.A.; Miroshnikov, A.I. Enzymatic transglycosylation of natural and modified nucleosides by immobilized thermostable nucleoside phosphorylases from Geobacillus stearothermophilus. Rus. J. Bioorg. Chem. 2009, 35, 739-745. [CrossRef]

16. Zhou, X.; Mikhailopulo, I.A.; Bournazou, M.N.; Neubauer, P. Immobilization of thermostable nucleoside phosphorylases on MagReSyn ${ }^{2}$ epoxide microspheres and their application for the synthesis of 2,6-dihalogenated purine nucleosides. J. Mol. Catal. B Enzym. 2015, 115, 119-127. [CrossRef]

17. Michailidou, F.; Lebl, T.; Slawin, A.M.Z.; Sharma, S.V.; Brown, M.J.B.; Goss, R.J.M. Fluorinated uridine analogues provide insight into a neighbouring-group participation mechanism. Molecules 2020, 25, 5513. [CrossRef] 
18. Miroshnikov, A.I.; Esipov, R.S.; Muravyova, T.I.; Konstantinova, I.D.; Fateev, I.V.; Mikhailopulo, I.A. A new strategy for the synthesis of nucleosides: One-pot enzymatic transformation of D-pentoses into nucleosides. Open Conf. Proc. J. 2010, 1, 98-102. [CrossRef]

19. Panosian, T.D.; Nannemann, D.P.; Watkins, G.R.; Phelan, V.V.; McDonald, W.H.; Wadzinski, B.E.; Iverson, T. M Bacillus cereus phosphopentomutase is an alkaline phosphatase family member that exhibits an altered entry point into the catalytic cycle. J. Biol. Chem. 2011, 286, 8043-8054. [CrossRef] [PubMed]

20. Taverna-Porro, M.; Bouvier, L.A.; Pereira, C.A.; Montserrat, J.M.; Iribarren, A.M. Chemoenzymatic preparation of nucleosides from furanoses. Tetrahedron Lett. 2008, 49, 2642-2645. [CrossRef]

21. Stepchenko, V.A.; Seela, F.; Esipov, R.S.; Miroshnikov, A.I.; Sokolov, Y.A.; Mikhailopulo, I.A. Enzymatic synthesis of $2^{\prime}$-deoxy- $\beta$-dribonucleosides of 8-azapurines and 8-aza-7-deazapurines. Synlett 2012, 23, 1541-1545.

22. Esipov, R.S.; Abramchik, Y.A.; Fateev, I.V.; Konstantinova, I.D.; Kostromina, M.A.; Muravyova, T.I.; Artemova, K.G.; Miroshnikov, A.I. A cascade of thermophilic enzymes as an approach to the synthesis of modified nucleotides. Acta Nat. 2016, 8, 161-168. [CrossRef]

23. Esipov, R.S.; Abramchik, Y.A.; Fateev, I.V.; Muravyova, T.I.; Scoblov, Y.S.; Kostromina, M.A.; Miroshnikov, A.I. Preparation and study of the substrate specificity of thermophilic ribokinase from Thermus sp. 2.9. Russ. J. Biopharm. 2016, 8, 3-12.

24. Timofeev, V.I.; Fateev, I.V.; Kostromina, M.A.; Abramchik, Y.A.; Konstantinova, I.D.; Volkov, V.V.; Lykoshin, D.D.; Mikheeva, O.O.; Muravieva, T.I.; Esipov, R.S.; et al. The comparative analysis of the properties and structures of purine nucleoside phosphorylases from thermophilic bacterium Thermus thermophilus HB27. J. Biomol. Struct. Dyn. 2020. [CrossRef] [PubMed]

25. Robins, M.J.; Uznański, B. Nucleic acid related compounds. 33. Conversions of adenosine and guanosine to 2, 6-dichloro, 2-amino-6-chloro, and derived purine nucleosides. Can. J. Chem. 1981, 59, 2601-2607. [CrossRef]

26. Robins, M.J.; Zou, R.; Hansske, F.; Madej, D.; Tyrrell, D.L.J. Synthesis, transformation chemistry, and biological activity of guanine nucleosides and analoges. Nucleosides Nucleotides 1989, 8, 725-741. [CrossRef]

27. Berzin, V.B.; Dorofeeva, E.V.; Leonov, V.N.; Miroshnikov, A.I. The preparative method for 2-fluoroadenosine synthesis. Rus. J. Bioorg. Chem. 2009, 35, 193-196. [CrossRef]

28. Chuvikovsky, D.V.; Esipov, R.S.; Skoblov, Y.S.; Chupova, L.A.; Muravyova, T.I.; Miroshnikov, A.I.; Lapinjoki, S.; Mikhailopulo, I.A. Ribokinase from E. coli: Expression, purification, and substrate specificity. Bioorg. Med. Chem. 2006, 14, 6327-6332. [CrossRef]

29. Esipov, R.S.; Gurevich, A.I.; Chuvikovsky, D.V.; Chupova, L.A.; Muravyova, T.I.; Miroshnikov, A.I. Overexpression of Escherichia coli genes encoding nucleoside phosphorylases in the pET/Bl21(DE3) system yields active recombinant enzymes. Protein Expr. Purif. 2002, 24, 56-60. [CrossRef] [PubMed]

30. Lowry, O.H.; Rosebrough, N.J.; Farr, A.L.; Randall, R.J. Protein measurement with the Folin phenol reagent. J. Boil. Chem. 1951, 193, 265-275. [CrossRef]

31. Laemmli, U.K. Cleavage of structural proteins during the assembly of the head of bacteriophage T4. Nature 1970, 227, 680-685. [CrossRef]

32. Konstantinova, I.D.; Fateev, I.V.; Miroshnikov, A.I. The arsenolysis reaction in the biotechnological method of synthesis of modified purine $\beta$-D-arabinonucleosides. Rus. J. Bioorg. Chem. 2016, 42, 372-380. [CrossRef]

33. Fateev, I.V.; Antonov, K.V.; Konstantinova, I.D.; Muravyova, T.I.; Seela, F.; Esipov, R.S.; Miroshnikov, A.I.; Mikhailopulo, I.A. The chemoenzymatic synthesis of clofarabine and related 2 -deoxyfluoroarabinosyl nucleosides: The electronic and stereochemical factors determining substrate recognition by E. coli nucleoside phosphorylases. Beilstein J. Org. Chem. 2014, 10, 1657-1669.

34. Bunton, C.A.; Hummers, E. The hydrolyses of $\alpha$-D-ribose and $\alpha$-D-glucose-1-phosphate. J.Org. Chem. 1969, 34, 572-576. [CrossRef]

35. Kamel, S.; Weiß, M.; Klare, H.F.T.; Mikhailopulo, I.A.; Neubauer, P.; Wagner, A. Chemo-enzymatic synthesis of $\alpha$-D-pentofuranose1-phosphates using thermostable pyrimidine nucleoside phosphorylases. Mol. Catal. 2018, 458, 52-59. [CrossRef]

36. Bennett, E.M.; Li, C.; Allan, P.W.; Parker, W.B.; Ealick, S.E. Structural Basis for Substrate Specificity of Escherichia coli Purine Nucleoside Phosphorylase. J. Biol. Chem. 2003, 278, 47110-47118. [CrossRef] [PubMed] 